\title{
The finitistic dimension of a Nakayama algebra.
}

\author{
Claus Michael Ringel
}

\begin{abstract}
Let $A$ be an artin algebra, Gélinas has introduced an interesting upper bound for the finitistic dimension fin-pro $A$ of $A$, namely the delooping level del $A$. We assert that fin-pro $A=\operatorname{del} A$ for any Nakayama algebra $A$. This yields also a new proof that the finitistic dimension of $A$ and its opposite algebra are equal, as shown recently by Sen. If $S$ is a simple module, let $e(S)$ be the minimum of the projective dimension of $S$ and of its injective envelope (one of these numbers has to be finite); and $e^{*}(S)$ the minimum of the injective dimension of $S$ and of its projective cover. Then the finitistic dimension of $A$ is the maximum of the numbers $e(S)$, as well as the maximum of the numbers $e^{*}(S)$.

Using suitable syzygy modules, we construct a permutation $h$ of the simple modules $S$ such that $e^{*}(h(S))=e(S)$. In particular, this shows for $z \in \mathbb{N}$ that the number of simple modules $S$ with $e(S)=z$ is equal to the number of simple modules $S^{\prime}$ with $e^{*}\left(S^{\prime}\right)=z$.
\end{abstract}

Key words. Nakayama algebra. Finitistic dimension. Delooping level. Desuspending level. The grade of a module. The homological permutation. Catalan combinatorics.

2020 Mathematics Subject classification. Primary 16G10, Secondary 16G20, 16G70, 16E05, $16 \mathrm{E} 10,16 \mathrm{E} 65$.

\section{Introduction.}

Let $A$ be an artin algebra. The modules $M$ to be considered are usually left $A$-modules. Often (but not always) we will assume in addition that $M$ is finitely generated. We denote by $A^{\text {op }}$ the opposite algebra of $A$ (and stress that the right $A$-modules are just the (left) $A^{\mathrm{op}}$-modules).

The projective dimension of a module $M$ will be denoted by $\operatorname{pd} M$, its injective dimension by id $M$. We write fin-pro $A$ for the supremum of the projective dimension of finitely generated left modules of finite projective dimension, and similarly, fin-inj $A$ for the supremum of the injective dimension of finitely generated left modules of finite injective dimension. Note that fin-pro $A$ is called the (small) finitistic dimension of $A$ and fin-inj $A$ is just the finitistic dimension of the opposite algebra $A^{\mathrm{op}}$. The finitistic dimension was considered first by Serre [S] in 1955. In 1960, its relevance was stressed by Bass [B]. It lies at the center of the classical homological conjectures.

We will focus the attention to Nakayama algebras: An algebra $A$ is called Nakayama provided all indecomposable modules are serial (that means: they have a unique composition series). The module category of a Nakayama algebra is well understood and many questions which are difficult to deal with in general, can easily be answered for Nakayama algebras. But, actually, only very recently, it was shown by Sen [S2] that fin-pro $A=$ fin-inj $A$ for a Nakayama algebra $A$. This seems to be an important observation. We will provide here a new proof as well as some further information on fin-pro $A$. 
Being interested in the finitistic dimension of an algebra $A$, Gélinas [Ge] has introduced a new invariant, the delooping level $\operatorname{del} A$. If $M$ is a module, let $\Omega M$ be its (first) syzygy module (it is the kernel of a projective cover $P M \rightarrow M$, and sometimes also called the "loop" module of $M$ ) and let $\Sigma M$ be its (first) suspension module (the cokernel of an injective envelope $M \rightarrow I M)$. The delooping level del $S$ of a simple module $S$ is the smallest number $d \geq 0$ such that $\Omega^{d} S$ is a direct summand of $P \oplus \Omega^{d+1} M$, where $M, P$ are finitely generated modules with $P$ projective (and $\operatorname{del} S=\infty$, if such a number $d$ does not exist). Similarly, one may define the desuspending level des $S$ of $S$ as the smallest number $d \geq 0$ such that $\Sigma^{d} S$ is a direct summand of $I \oplus \Sigma^{d+1} M$ for some finitely generated modules $M$, $I$ with $I$ injective (and $\operatorname{des} S=\infty$, if such a $d$ does not exist). By definition, $\operatorname{del} A$ is the maximum of $\operatorname{del} S$, where $S$ runs through the simple modules, and $\operatorname{des} A$ is the corresponding maximum of the numbers $\operatorname{des} S$. Of course, $\operatorname{des} A=\operatorname{del} A^{\mathrm{op}}$.

If $A$ is a Nakayama algebra, we will show that fin-pro $A=\operatorname{del} A$, thus, altogether

$$
\text { fin-pro } A=\text { fin-inj } A=\operatorname{del} A=\operatorname{des} A \text {. }
$$

The proof provides an explicit formula for the finitistic dimension of a Nakayama algebra $A$, namely

$$
\text { fin } \operatorname{pro} A=\max _{S} \min \{\operatorname{pd} S, \operatorname{pd} I S\}
$$

where $S$ runs through the simple modules (note that we will show that at least one of $\operatorname{pd} S, \operatorname{pd} I S$ is finite). It may happen that both $S$ and $I S$ have finite, but different, projective dimension, then the formula shows that we may delete $S$ on the right when looking for the maximum! Also, we may delete on the right all the torsionless simple modules (namely, if $S$ is torsionless and simple, then pd $I S=0$ ).

Let us write $e(S)=\min \{\operatorname{pd} S, \operatorname{pd} I S\}$ and $e^{*}(S)=\min \{\operatorname{id} S, \mathrm{id} P S\}$ for any simple module $S$, thus fin-pro $A=\max _{S} e(S)=\max _{S} e^{*}(S)$. We show in sections 4 and 5 that for any natural number $z$, there is a bijection $h_{z}$ between the set of simple modules $S$ with $e(S)=z$ and the set of simple modules $S^{\prime}$ with $e^{*}\left(S^{\prime}\right)=z$. These bijections $h_{z}$ can be combined in order to obtain a permutation $h$ of the simple modules. If $S$ is a simple module, then

$$
h(S)=h_{e(S)}(S)=\operatorname{top} \Omega^{e(S)} N S,
$$

where $N S=S$ if $e(S)$ is odd and $N S=I S$ if $e(S)$ is even (see also the description of $h$ in terms of $\tau, \phi$ and $\gamma$ mentioned in 4.4). We call $h=h_{A}$ the homological permutation for $A$.

Outline of the paper. Section 2 deals with arbitrary artin algebras. We will review the definition of the delooping level of a module, as introduced by Gélinas. We will show in 2.3: If $X$ is a submodule of a finitely generated module $Y$, then $\operatorname{del} X \leq \operatorname{pd} Y$. As a consequence, we get: if any simple module is a submodule of a finitely generated module of finite projective dimension, then $\operatorname{del} A \leq$ fin-pro $A$, see Theorem 2.4.

Sections 3 to 5 and the appendices restrict the attention to Nakayama algebras. Following Madsen [M], we show that if $A$ is Nakayama, then any simple module $S$ is a submodule of a finitely generated module of finite projective dimension, see 3.6. As a consequence, we can apply the general considerations of section 2, see 3.7. The assertions (1) to (8) in 3.7 may be considered as the heart of the first part of the paper. 
The second part of the paper, the sections 4 and 5, are devoted to the homological permutation $h$ of a Nakayama algebra. The simple modules $S$ with $e(S)=0$ are just the torsionless simple modules, and $h$ sends the socle of an indecomposable projective-injective module $B$ to the top of $B$. For $z \geq 1$, the bijection $h_{z}$ comes equipped with long exact sequences (the corresponding ties or bonds), namely minimal projective resolutions of $T$ or $I T$ and minimal injective coresolutions of $S$ or $P S$, where $h_{z}(T)=S$. As we will see in 4.4, the bijections $h_{z}$ can be described in terms of the Auslander-Reiten translations $\tau$ and $\tau^{-}$, and the functions $\operatorname{soc} P(-)$ and top $I(-)$.

There are several appendices which expand the discussion. In Appendix A, we consider the functions $\psi=\tau^{-}$top $I(-)$ and $\gamma=\tau \operatorname{soc} P(-)$ on the set of simple modules for a cyclic Nakayama algebra. We use covering theory and deal with corresponding monotone endofunctions of $\mathbb{Z}$. In this appendix $A$, we also will draw the attention to the resolutuion quiver and the coresolution quiver of a Nakayama algebra.

In Appendix B, we provide an outline of Sen's $\epsilon$-construction which allowed him to prove several interesting results using induction, for example the equality of the finitistic dimension of a Nakayama algebra $A$ and its opposite algebra [S2], but also, very recently, the equality fin-pro $A=\operatorname{del} A$, see [S3]. In the center of this approach lies the fact that there are canonical bijections between several sets of modules. All these sets have cardinality $r$, where $r$ is the minimal number of relations needed to define $A$. Appendix $\mathrm{C}$ is devoted to these bijections.

In the final Appendix D, we discuss two further invariants of a simple module $S$ which are related to the finitistic dimension, and which are considered by Gélinas, namely grade $S$ as well as the set of numbers $t$ such that $\mho^{t} \Omega^{t} S$ is torsionless.

Let us stress that the discussions in sections 3, 4 and 5 and in the Appendices A, $\mathrm{B}, \mathrm{C}$ are purely combinatorial. Of course, the terminology which we use comes from the theory of rings and modules, and concerns homological and categorical invariants. However, for a Nakayama algebra $A$, these invariants can be expressed in combinatorial terms, say starting from the Auslander-Reiten quiver of $A$ (this is a translation quiver). In particular, the homological permutation defined in 4.2 should be seen in the light of (Catalan) combinatorics, see 4.4.

Acknowledgment. The author wants to thank Vincent Gélinas, Emre Sen and Xiao$\mathrm{Wu}$ Chen as well as a referee for fruitful suggestions concerning the presentation of the results.

\section{The delooping level, the desuspending level.}

2.1. Definitions. Let $M$ be a finitely generated module. The delooping level del $M$ of $M$ is the minimal number $d \geq 0$ such that $\Omega^{d} M$ belongs to add ${ }_{A} A \oplus \Omega^{d+1} M^{\prime}$ for some finitely generated module $M^{\prime}$. (Note that if $\Omega^{d} M$ belongs to add ${ }_{A} A \oplus \Omega^{d+1} M^{\prime}$ for some module $M^{\prime}$, then $\Omega^{d+1} M$ belongs to add ${ }_{A} A \oplus \Omega^{d+2} M^{\prime}$.) This definition is due to Gélinas $[\mathrm{Ge}]$. As we have mentioned already, the delooping level of the algebra $A$ is defined as $\operatorname{del} A=\max _{S} \operatorname{del} S$, where $S$ are the simple modules.

There is the dual concept of the desuspending level des $M$ of a finitely generated module $M$, this is the minimal number $d \geq 0$ such that $\Sigma^{d} M$ belongs to add $D\left(A_{A}\right) \oplus \Sigma M^{\prime}$ 
for some finitely generated module $M^{\prime}$. Also, we put $\operatorname{des} A=\max _{S} \operatorname{del} S$, where $S$ are the simple modules. Clearly, $\operatorname{des} A=\operatorname{del} A^{\text {op }}$.

In addition, let Fin-pro $A$ be the supremum of $\operatorname{pd} N$, where $N$ has finite projective dimension, but is not necessarily finitely generated (Fin-pro $A$ is usually called the big finitistic dimension), as well as Fin-inj $A$, the supremum of id $N$, where $N$ has finite injective dimension, but not necessarily finitely generated.

2.2. Proposition (Gélinas). Let $M$ be a finitely generated module. If there is a (not necessarily finitely generated) module $N$ with finite injective dimension $d \geq 1$ such that $\operatorname{Ext}^{d}(M, N) \neq 0$, then $d \leq \operatorname{del} M$.

Proof. Let $N$ be a module with $d=\operatorname{id} N$ and $1 \leq d<\infty$. Then $\operatorname{Ext}^{d+1}(X, N)=0$ for all modules $X$. Let $M$ be a finitely generated module and assume that del $M<d$. By definition, $\Omega^{d-1} M$ belongs to add ${ }_{A} A \oplus \Omega^{d} M^{\prime}$, for some finitely generated module $M^{\prime}$. It follows that $\operatorname{Ext}^{d}(M, N)=\operatorname{Ext}^{1}\left(\Omega^{d-1} M, N\right)$ belongs to add $\operatorname{Ext}^{1}\left({ }_{A} A \oplus \Omega^{d} M^{\prime}, N\right)$, but

$$
\operatorname{Ext}^{1}\left({ }_{A} A \oplus \Omega^{d} M^{\prime}, N\right)=\operatorname{Ext}^{1}\left(\Omega^{d} M^{\prime}, N\right)=\operatorname{Ext}^{d+1}\left(M^{\prime}, N\right)=0,
$$

and therefore $\operatorname{Ext}^{d}(M, N)=0$, a contradiction.

Corollary: Fin-inj $A \leq \operatorname{del} A$.

Proof. We have to show: If $N$ is an arbitrary module with finite injective dimension $d$, then $d \leq \operatorname{del} S$ for some simple module $S$. This is clear for $d=0$. Thus, we can assume that $d \geq 1$. Since id $N=d$, there is a simple module $S$ with $\operatorname{Ext}^{d}(S, N) \neq 0$. The Proposition yields $d \leq \operatorname{del} S$.

\section{$2.2^{\prime}$. The dual assertions.}

Proposition. Let $M$ be a finitely generated module. If there is a (not necessarily finitely generated) module $N$ with finite projective dimension $d \geq 1$ which satisfies $\operatorname{Ext}^{d}(N, M) \neq 0$, then $d \leq \operatorname{des} M$.

Corollary: Fin-pro $A \leq \operatorname{des} A$.

2.3. Proposition. If $X$ is a submodule of a finitely generated module $Y$, then

$$
\operatorname{del} X \leq \operatorname{pd} Y \text {. }
$$

Proof. Let $\operatorname{pd} Y=d$. We can assume that $d<\infty$. We apply the horseshoe lemma to the exact sequence $0 \rightarrow X \rightarrow Y \rightarrow Y / X \rightarrow 0$ and obtain an exact sequence

$$
0 \rightarrow \Omega^{d} X \rightarrow P^{\prime} \oplus \Omega^{d} Y \rightarrow \Omega^{d}(Y / X) \rightarrow 0,
$$

where $P^{\prime}$ is projective. Since $\Omega^{d} Y$ is projective, we see that the middle term $E=P^{\prime} \oplus \Omega^{d} Y$ is projective and the projective cover $P\left(\Omega^{d}(Y / X)\right)$ of $\Omega^{d}(Y / X)$ is a direct summand of $E$, say $E=P^{\prime \prime} \oplus P\left(\Omega^{d}(Y / X)\right)$ for some projective module $P^{\prime \prime}$. Therefore

$$
\Omega^{d} X=P^{\prime \prime} \oplus \Omega\left(\Omega^{d}(Y / X)\right)=P^{\prime \prime} \oplus \Omega^{d+1}(Y / X) .
$$


This shows that del $X \leq d$.

Remark. The inequality

$$
\operatorname{del} X \leq \min \{\operatorname{pd} Y \mid Y \text { finitely generated and } X \subseteq Y\}
$$

may be proper. For example, if $A$ is representation-finite, and $X$ is any finitely generated module, then $\operatorname{del} X$ is, of course, finite. On the other hand, if $X$ is an injective module with infinite projective dimension, then $X \subseteq Y$ implies that also pd $Y$ is infinite (since $X$ is a direct summand of $Y$ ), thus the right hand side is $\infty$. A typical example of a representation finite algebra with an injective module with infinite projective dimension is the radical-square-zero algebra $A$ whose quiver has two vertices, say 1 and 2, and two arrows: a loop at 1 and an arrow $2 \rightarrow 1$. The simple module $S(2)$ is injective and has infinite projective dimension (since $\Omega S(2)=S(1)$ and $\Omega S(1)=S(1)$ ), Also, there are just five isomorphism classes of indecomposable modules.

\section{$2.3^{\prime}$. The dual assertion.}

Proposition. If $X$ is a factor module of a finitely generated module $Y$. Then

$$
\operatorname{des} X \leq \operatorname{id} Y \text {. }
$$

2.4. Theorem. Assume that every simple module $S$ is a submodule of a finitely generated module $M_{S}$ of finite projective dimension. Let $d=\max _{S} \operatorname{pd} M_{S}$. Then

$$
\text { Fin-inj } A \leq \operatorname{del} A \leq d \leq \text { fin-pro } A \text {. }
$$

Proof. The first inequality is Corollary 2.2. According to Proposition 2.3, we have $\operatorname{del} S \leq \operatorname{pd} M_{S}$, thus $\operatorname{del} A=\max _{S} \operatorname{del} S \leq \max \operatorname{pd} M_{S}=d$. Finally, we have of course $\operatorname{pd} M_{S} \leq$ fin-pro $A$, thus $d=\max \operatorname{pd} M_{S} \leq$ fin-pro $A$.

Recall that an algebra is called left Kasch provided any simple module occurs as a left ideal. Thus, $A$ is left Kasch if and only if any simple module is a submodule of a module of projective dimension $d=0$. The case $d=0$ of 2.4 is therefore just the well-known assertion that Fin-inj $A=0$ for any Kasch algebra $A$.

\section{4'. The dual assertion.}

Theorem. Assume that every simple module $S$ is a factor module of a finitely generated module $N_{S}$ of finite injective dimension. Let $d^{\prime}=\max _{S}$ id $N_{S}$. Then

$$
\text { Fin-pro } A \leq \operatorname{des} A \leq d^{\prime} \leq \text { fin-inj } A \text {. }
$$

2.5. Combination. Assume that every simple module $S$ is a submodule of a finitely generated module $M_{S}$ of finite projective dimension and also a factor module of a finitely generated module $N_{S}$ of finite injective dimension. Let $d=\max _{S} \operatorname{pd} M_{S}$, and $d^{\prime}=$ $\max _{S}$ id $N_{S}$. Then

$$
\text { fin-pro } A=\text { fin-inj } A=\text { Fin-pro } A=\text { Fin-inj } A=\operatorname{del} A=\operatorname{des} A=d=d^{\prime} .
$$


2.6. Remark. Clearly, 2.5 applies to a large class of algebras, but the remaining parts of the paper are devoted just to Nakayama algebras. As one of the referees has stressed, 2.5 can be used to obtain a direct proof of the following result (see Gélinas [G1] , 1.18); it concerns the so called Gorenstein algebras of Gorenstein dimension $d$ :

Let $A$ be an artin algebra with minimal injective cogenerator $Q$. Assume that $\operatorname{pd} Q=$ $d<\infty$ and $\operatorname{id}_{A} A=d^{\prime}<\infty$. Then

$$
\text { fin-pro } A=\text { fin-inj } A=\text { Fin-pro } A=\text { Fin-inj } A=\operatorname{del} A=\operatorname{des} A=d=d^{\prime} .
$$

Namely, every simple module $S$ is a submodule of $M_{S}=Q$ and a factor module of $N_{S}=$ ${ }_{A} A$. By assumption, pd $Q=d$ and id ${ }_{A} A=d^{\prime}$.

\section{Nakayama algebras.}

Let $A$ be a Nakayama algebra. Let us note that if $M$ is an indecomposable module, then $\Omega M$ and $\Sigma M$ are either zero or indecomposable again. An indecomposable module $M$ of finite projective dimension will be said to be even, or odd provided its projective dimension is even, or odd, respectively.

A connected Nakayama algebra $A$ is called linear provided there is a simple projective module, otherwise $A$ is called cyclic.

3.1. The maps $\psi$ and $\gamma$. We denote by $D \operatorname{Tr}$ and $\operatorname{Tr} D$ the Auslander-Reiten translations in $\bmod A$. If $M$ is indecomposable and not projective, we write $\tau M=D \operatorname{Tr} M$; if $M$ is indecomposable and not injective, we write $\tau^{-} M=\operatorname{Tr} D M$ (let us stress that we apply $\tau$ only to modules which are indecomposable and not projective, and $\tau^{-}$only to modules which are indecomposable and not injective).

If $S$ is a simple module, let $\psi S=\tau^{-}$top $I S$ provided top $I S$ is not injective. There is the dual concept: If $S$ is a simple module, let $\gamma S=\tau \operatorname{soc} P S$ provided $\operatorname{soc} P S$ is not projective. [The functions $\psi$ and $\gamma$ are important tools for dealing in particular with cyclic Nakayama algebras; their study goes back to Gustafson $[\mathrm{Gu}]$. They were used in many papers. The appendix A of this paper will focus the attention to further properties of $\psi$ and $\gamma$. In particular, one can define the coresolution quiver (or $\psi$-quiver) of a cyclic Nakayama algebra $A$. The $\psi$-paths considered in 3.4 are just the paths in the coresolution quiver. Similarly, using $\gamma$, we obtain the resolution quiver (or $\gamma$-quiver) of a cyclic Nakayama $A$.]

Lemma. (a) $A$ simple module $U$ is in the image of $\psi$ if and only if $\operatorname{pd} U \geq 2$. A simple module $T$ is in the image of $\gamma$ if and only if $\operatorname{id} T \geq 2$.

(b) If $M$ is an indecomposable module, and $m \in \mathbb{N}$. Then either $\mathrm{pd} M<2 m$ or else top $\Omega^{2 m} M=\gamma^{m} \operatorname{top} M$. Similarly, either id $M<2 m$ or else $\operatorname{soc} \Sigma^{2 m} M=\psi^{m} \operatorname{soc} M$.

Proof. (a) We show the second assertion (the first follows by duality).

Let $T$ be simple with id $T \geq 2$. Then $\Sigma T$ is not injective, thus we have a proper inclusion $\Sigma T \subset I \Sigma T$. We denote by $S$ the top of $I \Sigma T$. Now all the indecomposable modules $M$ which properly include $\Sigma T$ have to be projective. In particular, $I \Sigma T$ is projective and therefore $I \Sigma T=P S$. Since $\operatorname{soc} P S=\operatorname{soc} \Sigma T$ is not projective, we can apply $\tau$. By definition, $\gamma S=\tau \operatorname{soc} P S=\tau \operatorname{soc} I \Sigma T=\tau \tau^{-} T=T$. This shows that $T$ is in the image of $\gamma$. 
Conversely, let $S$ be any simple module and $T=\gamma S$. We want to show that $\Sigma T$ is not injective. Now $\Sigma T$ and $P S$ have the same socle, thus both are submodules of the projective module $I P S$. Since $I P S$ is serial, its submodules are pairwise comparable. If $P S \subseteq \Sigma T$, then $I T$ has a submodule $X$ of length $|P S|+1$ which maps onto $P S$. But this is impossible since $P S$ is projective. It follows that there is the proper inclusion $\Sigma T \subset P S$. This shows that $\Sigma T$ is not injective, thus id $T \geq 2$.

(b) See [R2], section 3, Corollary.

3.2. Nakayama algebras have been investigated quite thoroughly by Madsen $[\mathrm{M}]$. We will use many of his results, in particular:

Lemma (Madsen). Let $A$ be any Nakayama algebra and $M$ an indecomposable module.

(a) If $X$ is a subfactor of $M$, and $M$ is odd, then $X$ is odd and $\operatorname{pd} X \leq \operatorname{pd} M$.

(b) If $X$ is a subfactor of $M$, and $X$ is even, then $M$ is even and $\operatorname{pd} M \leq \operatorname{pd} X$.

(c) A simple module $S^{\prime}$ is a composition factor of $\Omega^{2} S$ if and only if $\psi\left(S^{\prime}\right)=S$ (and then the multiplicity of $S^{\prime}$ in $\Omega^{2} S$ is 1$)$.

Proof. For (a) and (b), see $[\mathrm{M}], 2.2$. For (c), see $[\mathrm{M}] 3.1$ (note that the assumption that $A$ is a cyclic Nakayama algebra is not needed).

Corollary. Let $X \subseteq Y$ be indecomposable modules. If $Y$ is odd, then $\operatorname{pd} X \leq \operatorname{pd} Y$. If $Y$ is even, then $\operatorname{pd} I X \leq \operatorname{pd} Y$.

Proof. The first assertion is a special case of (a). Thus, assume that $Y$ is even. We look at the inclusion $Y \subseteq I Y=I X$ and use (b): it asserts that $\operatorname{pd} I X \leq \operatorname{pd} Y$.

3.3. Maximum property of odd modules. Let $A$ be any Nakayama algebra. An indecomposable module $M$ is odd if and only if all composition factors of $M$ are odd; and then $\operatorname{pd} M$ is the maximum of $\operatorname{pd} S$, where $S$ is a composition factor of $M$.

Proof. Let $M$ be indecomposable. First, assume that $M$ is odd. According to 3.2 (a), all composition factors $S$ of $M$ are odd and $\operatorname{pd} S \leq \operatorname{pd} M$. Of course, at least one of the composition factors must have $\operatorname{pd} S=\operatorname{pd} M$ (since the class of modules of projective dimension smaller $\operatorname{pd} M$ is closed under extensions). Thus $\operatorname{pd} M$ is odd. Conversely, assume that all composition factors of $M$ are odd. According to [M] Proposition 4.1, it follows that $\operatorname{pd} M$ is the maximum of $\operatorname{pd} S$, where $S$ is a composition factor of $M$.

Remark. There is the following consequence: Let $A$ be a Nakayama algebra. For any odd number $1 \leq i \leq$ fin-pro $A$, there is a simple module $S$ with $\mathrm{pd} S=i$. (Note that the odd simple modules of a cyclic Nakayama algebra are nicely displayed in the $\psi$-quiver; see Appendix A.8 and Proposition 3.5.)

Proof. There is a (finitely generated) module $M$ with $\operatorname{pd} M=d=$ fin-pro $A$. Thus, for any number $0 \leq i \leq d$, there is an indecomposable module $M_{i}$ with $\operatorname{pd} M_{i}=i$, namely a direct summand of $\Omega^{d-i} M$. If $i$ is odd, 3.3 asserts that $M_{i}$ has a composition factor $S$ with $\operatorname{pd} S=i$.

The next two subsections 3.4 and 3.5 are devoted to cyclic Nakayama algebras. 
3.4. The $\psi$-paths (and the $\gamma$-paths). Let $\Lambda$ be a cyclic Nakayama algebra. A $\psi$-path of cardinality $m$ is a sequence $\left(S_{1}, S_{2}, \ldots, S_{m}\right)$ of simple modules with $S_{i+1}=\psi S_{i}$ for $1 \leq i<m$, it starts in $S_{1}$ and ends in $S_{m}$. If $\psi(S)=T$, we say that $S$ is a $\psi$-predecessor of $T$, If $\gamma(S)=T$, we say that $S$ is a $\gamma$-predecessor of $T$, Similarly, a $\gamma$-path of cardinality $m$ is a sequence $\left(S_{1}, S_{2}, \ldots, S_{m}\right)$ of simple modules with $S_{i+1}=\gamma S_{i}$ for $1 \leq i<m$, it starts in $S_{1}$ and ends in $S_{m}$.

For $A$ a cyclic Nakayama algebra, and $S$ a simple module, we define $a(S)$ and $a^{\prime}(S)$ as follows: Let $a(S)$ be the supremum of the cardinality of the $\psi$-paths ending in $S$, and dually, let $a^{\prime}(S)$ be the supremum of the cardinality of the $\gamma$-paths ending in $S$. Thus, $a(T)$ is the maximum of $1+a(S)$ with $S$ a $\psi$-predecessor of $T$ and $a^{\prime}(T)$ is the maximum of $1+a^{\prime}(S)$ with $S$ a $\gamma$-predecessor of $T$.

We say that a simple module $S$ is $\psi$-cyclic provided $a(S)=\infty$, or, equivalently, provided there is some number $e \geq 1$ with $\psi^{e} S=S$. Similarly, $S$ is $\gamma$-cyclic provided $a^{\prime}(S)=\infty$, thus provided there is some number $e \geq 1$ with $\gamma^{e} S=S$.

Finally, let $a(A)$ be the maximum of $a(S)$, where $S$ is simple and not $\psi$-cyclic. Note that $a(A)$ is also the maximum of $a^{\prime}(S)$, where $S$ is simple and not $\gamma$-cyclic, see A.1 in the Appendix.

If $a(A)=0$, then all simple modules are torsionless, thus all modules are torsionless, thus $A$ is selfinjective. For the topics discussed in the paper, we usually may assume that $A$ is not self-injective.

3.5. Proposition. Let $A$ be a cyclic Nakayama algebra, and $S$ a simple module.

(1) We have a $(S)$ finite if and only if $\operatorname{pd} S$ is odd, and then $\operatorname{pd} S=2 a(S)-1$, in particular, $\operatorname{pd} S<2 a(S)$. If $a(S)$ is infinite, then $\operatorname{pd} I S$ is even and $\operatorname{pd} I S \leq 2 a(A)$.

(2) Similarly, $a^{\prime}(S)$ finite if and only if id $S$ is odd, and then id $S=2 a^{\prime}(S)-1$, in particular, id $S<2 a(S)$. If $a^{\prime}(S)$ is infinite, then id $P S$ is even and id $P S \leq 2 a(A)$.

Proof. First, we show: If $a(S)$ finite, then $\mathrm{pd} S=2 a(S)-1$. We use induction on $a(S)$. If $a(S)=1$, then $S$ has no $\psi$-predecessor, thus $\operatorname{rad} P S$ is projective, thus $\operatorname{pd} S=1$. Now assume that $a(S) \geq 2$. Then $S$ has a $\psi$-predecessor, thus $\operatorname{rad} P S$ is not projective, and therefore pd $P S \geq 2$. As a consequence, $\Omega^{2} S \neq 0$. According to 3.2 (c), the composition factors of $\Omega^{2} S$ are the $\psi$-predecessors $S^{\prime}$ of $S$. If $S^{\prime}$ is a $\psi$-predecessor of $S$, then $a\left(S^{\prime}\right) \leq$ $a(S)-1$, and by induction pd $S^{\prime}=2 a\left(S^{\prime}\right)-1 \leq 2 a(S)-3$. And there is a predecessor $S^{\prime \prime}$ of $S$ with $a\left(S^{\prime \prime}\right)=a(S)-1$, thus with pd $S^{\prime \prime}=2 a\left(S^{\prime \prime}\right)-1=2 a(S)-3$. This shows that the composition factors of $\Omega^{2} S$ have odd projective dimension with maximum $2 a(S)-3$. The maximum property 3.3 asserts that $\operatorname{pd} \Omega^{2} S=2 a(S)-3$. Thus, pd $S=2+\operatorname{pd} \Omega^{2} S=$ $2 a(S)-1$.

Conversely, let us show: If pd $S$ is odd, then $a(S)$ is finite. The proof is again by induction. If $\operatorname{pd} S=1$, then $S$ cannot have a $\psi$-predecessor, thus $a(S)=1$. If $\operatorname{pd} S$ is odd and not 1, then $\operatorname{pd} \Omega^{2} S=\operatorname{pd} S-2$ is odd, again. Let $S^{\prime}$ be a predecessor of $S$. By 3.2 (c), $S^{\prime}$ is a composition factor of $\Omega^{2} S$. By the maximum property $3.3, \operatorname{pd} S^{\prime}$ is odd and $\operatorname{pd} S^{\prime} \leq \operatorname{pd} \Omega^{2} S=\operatorname{pd} S-2$. By induction, $a\left(S^{\prime}\right)$ is finite. Since $a\left(S^{\prime}\right)$ is finite for all predecessors $S^{\prime}$ of $S$, also $a(S)$ is finite.

Now assume that $a(S)$ is infinite. If $I S$ is projective, then its projective dimension is zero, therefore even. Thus, we suppose that $I S$ is not projective and show that $\Omega I S$ has odd projective dimension. The following picture displays part (of the universal covering) 
of the Auslander-Reiten quiver of $A$, with arrows going from left to right:

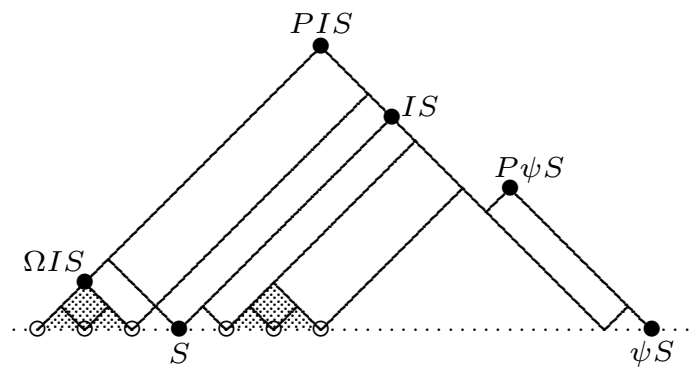

On the left and on the right of $S$ (at the lower boundary of he shaded areas) are the $\psi$ predecessors $S^{\prime}$ of $S$ which are different from $S$. For these modules $S^{\prime}$, we have $a\left(S^{\prime}\right)<\infty$, thus, as we have seen already, they have odd projective dimension. The module $\Omega I S$ has a filtration using such modules, thus 3.3 asserts that $\mathrm{pd} \Omega I S$ is odd, therefore $\mathrm{pd} I S$ is even. Since pd $\Omega I S \leq 2 a(A)-1$, we have pd $I S \leq 2 a(A)$. This completes the proof of $(1)$.

The assertions (2) follow by duality.

3.6. Theorem. Let $A$ be any Nakayama algebra and $S$ a simple module. Then $S$ is odd or IS is even. (Thus, at least one of $S, I S$ has finite projective dimension.)

Proof. We can assume that $A$ is connected. We can assume that $S$ is not odd, thus $\operatorname{pd} S$ is even or infinite. If pd $S$ is even, then Lemma 3.2 (b) asserts that pd $I S$ is even. If $\operatorname{pd} S$ is infinite, then $A$ has to be cyclic, and 3.5 (a) asserts first that $a(S)$ cannot be finite and then that $\operatorname{pd} I S$ is even.

Dual assertion. Let $A$ be any Nakayama algebra and $S$ a simple module. Then id $S$ is odd or id $P S$ is even. (Thus, at least one of $S, P S$ has finite injective dimension.)

\subsection{Summary.}

(1) Let $A$ be a Nakayama algebra. Then any simple module is a submodule of an indecomposable module with finite projective dimension, and a factor module of an indecomposable module with finite injective dimension.

Proof. Let $S$ be simple. Theorem 3.6 asserts that pd $S$ or pd $I S$ is finite, thus $S$ is a submodule of an indecomposable module with finite projective dimension. By duality, $S$ or $P S$ has finite injective dimension, thus $S$ is a factor module of an indecomposable module with finite injective dimension.

(2) Theorem. Let $A$ be a Nakayama algebra. If $S$ is simple, let $M_{S}=S$ provided $S$ has finite projective dimension, and $M_{S}=I S$ otherwise, and let $N_{S}=S$ provided $S$ has finite injective dimension, and $N_{S}=P S$ otherwise. Let $d=\max _{S} \operatorname{pd} M_{S}$ and $d^{\prime}=\max _{S}$ id $N_{S}$. Then

$$
\text { fin-pro } A=\operatorname{fin}-\operatorname{inj} A=\operatorname{del} A=\operatorname{des} A=d=d^{\prime} \text {. }
$$

Proof. We need to know that all the modules $M_{S}$ have finite projective dimension, and all the modules $P S$ have finite injective dimension. Then we can apply 2.5. Theorem 
3.6 and its dual assert that all the modules $M_{S}$ have finite projective dimension and that all the modules $N_{S}$ have finite injective dimension.

(3) Let $A$ be a Nakayama algebra and $X$ an indecomposable module. let us write

$$
e(X)=\min \{\operatorname{pd} X, \operatorname{pd} I X\}
$$

(a) We have

$$
e(X)=\min \{\operatorname{pd} Y \mid Y \text { finitely generated and } X \subseteq Y\}
$$

and

$$
\operatorname{del} X \leq e(X)
$$

(b) If $S$ is simple, then

$$
e(S)<\infty
$$

Proof. (a) Let $X \subseteq Y$, where $Y$ is finitely generated and pd $Y$ minimal. Since $X$ has simple socle, we can assume that $Y$ is indecomposable. Namely, let $Y=\bigoplus Y_{i}$ with all $Y_{i}$ indecomposable. Then $X$ is a submodule of some $Y_{i}$ and $\operatorname{pd} Y_{i} \leq \operatorname{pd} Y$.

If $Y$ is odd, then $X \subseteq Y$ shows that $\mathrm{pd} X \leq \operatorname{pd} Y$, see 3.2 (a). Thus the minimality of $\operatorname{pd} Y$ yields pd $X=\operatorname{pd} Y$. If $Y$ is even, then $Y \subseteq I Y=I X$ shows that $\operatorname{pd} I X \leq \operatorname{pd} Y$, see 3.2 (b). Thus, the minimality of $\operatorname{pd} Y$ yields $\operatorname{pd} I X=\operatorname{pd} Y$. The inequality $\operatorname{del} X \leq e(X)$ has been shown in 2.3.

(b) For the assertion $e(S)<\infty$, see Theorem 3.6.

Since $e(X)=\min \{\operatorname{pd} Y \mid Y$ finitely generated and $X \subseteq Y\}$, Gélinas has suggested to call the number $e(X)$ the embedding projective dimension of $X$.

(4) Theorem. Let $A$ be a Nakayama algebra. Then

$$
\text { fin } \operatorname{pro} A=\max _{S} e(S)
$$

were $S$ runs through the simple modules.

Proof. As we have mentioned in (3), we have del $X \leq e(X)$ for all modules $X$. Thus $\operatorname{del} A=\max _{S} \operatorname{del} S \leq \max _{S} e(S)$. If $S$ is simple, then, according to (1), pd $S$ or pd $I S$ is finite, thus $e(S)=\min \{\operatorname{pd} S, \operatorname{pd} I S\} \leq$ fin-pro $A$. This shows that

$$
\text { fin-pro } A=\operatorname{del} A=\max _{S} \operatorname{del} S \leq \max _{S} e(S) \leq \text { fin-pro } A
$$

(5) We can rewrite the definition of $e(S)$ as follows:

$$
e(S)=\left\{\begin{array}{cl}
\min \{\operatorname{pd} S, \operatorname{pd} I S\} \\
\operatorname{pd} S \\
\operatorname{pd} I S
\end{array} \quad \text { if } \quad \begin{array}{l}
S \text { is odd } I S \text { is not even, } \\
S \text { is not odd }
\end{array}\right.
$$


Note that if $I S$ is not even, then $S$ has to be odd, see Theorem 3.6, thus the definition covers all possible cases for the pair $(S, I S)$ and is well-defined.

Proof. Let $S$ be simple. If $I S$ is not even, then $\operatorname{pd} S \leq \operatorname{pd} I S$ (this is clear, if pd $I S=\infty$, whereas for $I S$ odd, we use the first part of Corollary 3.2), therefore pd $S=$ $\min \{\operatorname{pd} S, \operatorname{pd} I S\}=e(S)$. If $S$ is not odd, then $\operatorname{pd} I S \leq \operatorname{pd} S$ (this is clear, if $\operatorname{pd} S=\infty$, whereas. if $S$ is even, then we use the second part of Corollary 3.2). Therefore pd $I S=$ $\min \{\operatorname{pd} S, \operatorname{pd} I S\}=e(S)$.

Corollary 1. Let $A$ be a Nakayama algebra. If $S$ is simple, let

$$
f(S)=\left\{\begin{array}{cl}
0 & S \text { is odd, } I S \text { is even, } \\
\operatorname{pd} S & \text { if } \quad \begin{array}{l}
I S \text { is not even, } \\
\operatorname{pd} I S
\end{array} \quad S \text { is not odd }
\end{array}\right.
$$

Then $f(S)=e(S)$ for all simple modules $S$ such that $I S$ is not even or $S$ is not odd, thus $f(S) \leq e(S)$ for all simple modules $S$. Also,

$$
\text { fin-pro } A=\max _{S} f(S) \text {. }
$$

Proof of Corollary 1. As we have seen at the beginning of (5), $f(S)=e(S)$ if $I S$ is not even or $S$ is not odd. If $S$ is odd and $I S$ is even, then $f(S)=0 \leq \min \{\operatorname{pd} S, \operatorname{pd} I S\}=e(S)$. It follows that $f(S) \leq e(S)$ for all simple modules $S$.

In order to show that fin pro $A=\max _{S} f(S)$, we note that on the right hand side of the formula (4), we may delete all the terms $\min \{\operatorname{pd} S, \operatorname{pd} I S\}$, where $S$ is a simple module which is odd such that $I S$ is even. Namely, if $S$ is odd and $I S$ is even, then pd $S \neq \operatorname{pd} I S$, thus $\min \{\operatorname{pd} S, \operatorname{pd} I S\}<\max \{\operatorname{pd} S, \operatorname{pd} I S\} \leq$ fin-pro $A$.

Corollary 2. Let $A$ be a Nakayama algebra and $z=$ fin-pro $A$. If $z$ is odd, then there is $S$ simple with $\operatorname{pd} S=z$ and $\operatorname{pd} I S$ odd or infinite. If $z$ is even, then there is $S$ simple with $\operatorname{pd} I S=z$ and $\operatorname{pd} S$ even or infinite.

The proof follows from Corollary 1 and Theorem 3.6 as follows. If all simple modules are odd, then all non-zero modules are odd by the maximum principle, but this is impossible, since the projective modules are even. Thus fin-pro $A=\operatorname{pd} S$ for some $S$ with $I S$ not even (we call it the first case), or fin-pro $A=\operatorname{pd} I S$ for some $S$ with $S$ not odd (the second case). In the first case, pd $I S$ is odd or infinite. Also, since $I S$ is not even, $S$ has to be odd, thus $z=\operatorname{pd} S$ is odd, according to 3.6. In the second case, $\operatorname{pd} S$ is even or infinite. Since $S$ is not odd, 3.6 asserts that $z=\operatorname{pd} I S$ is even. Conversely, of course, if $z$ is odd, we cannot be in the second case, thus we are in the first case. And if $z$ is even, then we have to be in the second case.

(6) (Madsen). Let $A$ be a connected Nakayama algebra. If there exists a simple module $S$ such that $S$ is even or $I S$ is odd, then $A$ has finite global dimension.

Proof. If $\operatorname{pd} S$ is even, then see Madsen [M], Theorem 3.1. Thus assume that $I S$ is odd. Then all factor modules of $I S$ are odd, according to 3.2 (a). Assume that $X$ is a non-zero factor module of $I S$ which is not injective, thus there is an inclusion $X \subset Y$ 
with $Y / X$ simple. Then $Y$ is projective and $\operatorname{pd} Y / X=1+\operatorname{pd} X$ is even. Thus, we haven a simple module of even projective dimension and as mentioned already, $A$ has finite global dimension. We therefore may assume that all factor modules of $I S$ are injective. In particular, the simple factor module of $I S$ is injective, thus $A$ is a linear Nakayama algebra and thus has finite global dimension.

Corollary. Let $A$ be a connected Nakayama algebra with infinite global dimension. Let $z=$ fin-pro $A$. If $z$ is odd, then there is $S$ simple with $\operatorname{pd} S=z$ and $\operatorname{pd} I S$ infinite. If $z$ is even, then there is $S$ simple with $\operatorname{pd} I S=z$ and $\operatorname{pd} S$ infinite.

Proof: This follows from (6) and Corollary 2 in (5). Namely, Since $A$ has infinite global dimension, pd $I S$ cannot be odd and $\operatorname{pd} S$ cannot be even.

(7) Looking at the finitistic dimension of an algebra, the algebras of infinite global dimension are those of real interest. For algebras of infinite global dimension, the function $f$ defined in (5) can be defined slightly different:

Let $A$ be a connected Nakayama algebra with infinite global dimension. Then the function $f$ considered in (5) coincides with the following function $g$ :

$$
g(S)=\left\{\begin{array}{cl}
0 & \operatorname{pd} S, \operatorname{pd} I S \text { both are finite } \\
\operatorname{pd} S & \text { if } \quad \\
\operatorname{pd} I S & \operatorname{pd} I S \text { is infinite, }
\end{array}\right.
$$

(according to Theorem 3.6, at least one of $\operatorname{pd} S$ and $\mathrm{pd} I S$ is finite).

Corollary. Let $A$ be a connected Nakayama algebra with infinite global dimension. Then

$$
\text { fin-pro } A=\max _{S} g(S) \text {. }
$$

Remark. If $A$ has finite global dimension, then the function $g$ is the zero function. Thus, in this case, $g$ cannot be used for calculating fin-pro $A$ (fin-pro $A$ is just the global dimension of $A$, and the global dimension of a cyclic Nakayama algebra is at least 2).

Using (6), we obtain a short proof for the following result ([S2], Corollary 4.13):

(8) (Sen) Let $A$ be a connected Nakayama algebra which is Gorenstein and has infinite global dimension. Then fin-pro $A$ is even.

Proof. If fin-pro $A$ is odd, the Corollary in (6) provides an indecomposable injective module $I$ with pd $I$ infinite, a contradiction to the assumption that $A$ is Gorenstein.

3.8. Examples. The possible pairs ( $\operatorname{pd} S, \operatorname{pd} I S$ ). We have seen in 3.2: (a) if $I S$ is odd, then $S$ is odd and $\operatorname{pd} S \leq \operatorname{pd} I S$; (b) if $S$ is even, then $I S$ is even and $\operatorname{pd} S \geq$ pd $I S$. Theorem 3.6 shows that it is impossible that both $S$ and $I S$ have infinite projective dimension. All the remaining possibilities do occur. Here are some examples (for a general discussion, we refer to section 4.7).

$\star \operatorname{pd} S<\operatorname{pd} I S$, both odd: see $S=S_{7}$.

$\star \operatorname{pd} S=\operatorname{pd} I S$, both odd: see $S=S_{6}$. 
- $\operatorname{pd} S$ odd, $\operatorname{pd} I S=\infty$ : see $S=S_{3}$.

- $\operatorname{pd} S<\operatorname{pd} I S$, with pd $S$ odd, pd $I S$ even; see $S=S_{8}$ and $S=S_{10}$.

- $\operatorname{pd} S>\operatorname{pd} I S$, with $\operatorname{pd} S$ odd, $\operatorname{pd} I S$ even: see $S=S_{1}$ and $S=S_{9}$.

- $\operatorname{pd} S=\infty, \operatorname{pd} I S$ even: see $S=S_{4}$.

$\star \operatorname{pd} S>\operatorname{pd} I S$, both even: see $S=S_{5}$.

$\star \operatorname{pd} S=\operatorname{pd} I S$, both even: see $S=S_{2}$.

According to $3.7(6)$, the cases marked by a star $\star$ can occur only if $A$ has finite global dimension. For the two cases which occur both for finite and for infinite global dimension, we have provided two examples.

The following pictures exhibit Auslander-Reiten quivers (the Auslander-Reiten quiver of a cyclic Nakayama algebra lives on a cylinder — the dashed lines left and right have to be identified). A vertex of an Auslander-Reiten quiver is the isomorphism class $[M]$ of an indecomposable module $M$. But instead of drawing the vertex $[M]$, we insert here the corresponding value pd $M$ (this is a natural number or $\infty$ ). The lower boundary of the Auslander-Reiten quiver of a Nakayama algebra consists of the simple modules: those which are of interest here, are encircled and labeled $S_{1}, \ldots, S_{10}$.
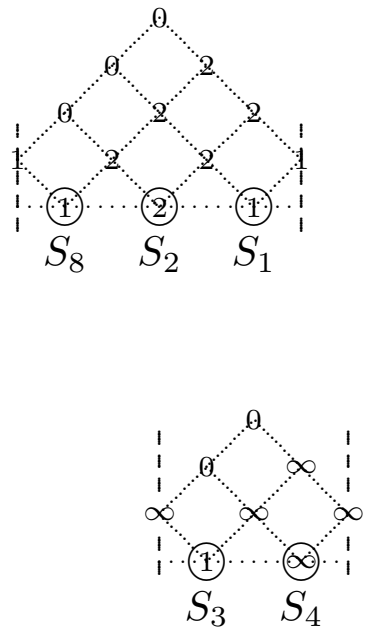
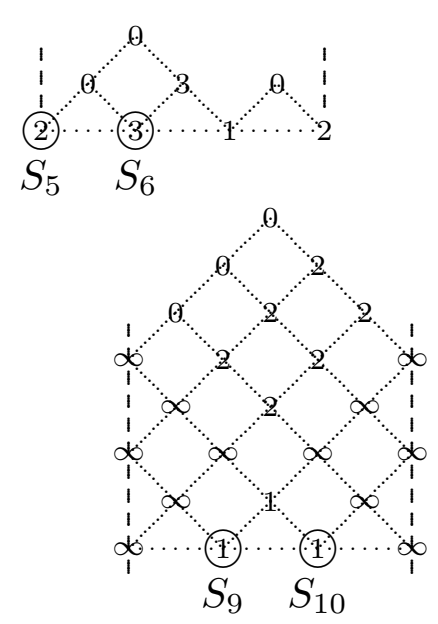

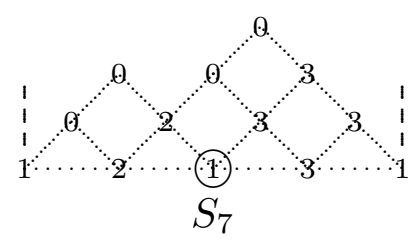

As we have mentioned, if $S$ is $\psi$-cyclic, then $\operatorname{pd} S \geq \operatorname{pd} I S$. But $\operatorname{pd} S \geq \operatorname{pd} I S$ may also happen for $S$ odd (and then pd $S>\operatorname{pd} I S$ ). It happens, of course, in case $S$ is torsionless, since then $\operatorname{pd} S>0$ and $\operatorname{pd} I S=0$. Here is an example of $S$, with $\operatorname{pd} S=3, \operatorname{pd} I S=2$, thus $S$ is odd, not torsionless, and $\operatorname{pd} S>\operatorname{pd} I S$.

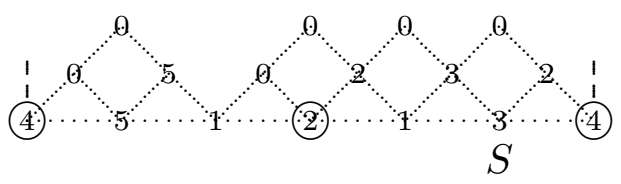

In addition, we see the following: Proposition 3.5 asserts that if $a(S)$ is infinite, then pd $I S$ is even, but the converse does not hold: $\operatorname{pd} I S$ may be even, whereas $a(S)$ is finite, as the examples $S_{1}$ and $S_{8}$ show. 
3.9. Remarks. We do not know whether the inequality del $S \leq e(S)$ can be proper. But there is always a simple module $S$ with $\operatorname{del} S=$ fin-pro $A$, and if $\operatorname{del} S=$ fin-pro $A$, then $\operatorname{del} S=e(S)$. Proof. The first assertion follows directly from $\max _{S} \operatorname{del} S=$ fin-pro $A$. If $\operatorname{del} S=$ fin-pro $A$, then the inequalities

$$
\operatorname{del} S \leq e(S) \leq \text { fin-pro } A
$$

yield the equality $\operatorname{del} S=\min \{\operatorname{pd} S, \operatorname{pd} I S\}$.

One also may compare $f(S)$ as introduced in $3.7(5)$ with del $S$. For the simple module $S_{8}$ mentioned in 3.8, we have $f\left(S_{8}\right)=0$, since $S_{8}$ is odd and $I S_{8}$ is even. On the other hand, since $S_{8}$ is not torsionless, del $S_{8} \geq 1$ (since pd $S_{8}=1$, we have del $S_{8}=1$ ).

3.10. Proposition. Let $A$ be a cyclic Nakayama algebra. Then

$$
2 a(A)-1 \leq \text { fin-pro } A \leq 2 a(A) .
$$

Proof. In order to show that $2 a(A)-1 \leq$ fin-pro $A$, take a simple module $S$ with $a(S)=a(A)$. According to $3.5(1)$, we have pd $S=2 a(S)-1=2 a(A)-1$.

For the second inequality, let $d=$ fin-pro $A$. There is a module $M$ with $\operatorname{pd} M=d$. If $d$ is odd, then 3.3 yields a composition factor $S$ of $M$ with pd $S=d$. According to 3.5 (1), we have $\operatorname{pd} S=2 a(S)-1$. Therefore $d=\operatorname{pd} S=2 a(S)-1 \leq 2 a(A)-1<2 a(A)$.

If $d$ is even and at least 2, then pd $\Omega M=d-1$ and 3.3 yields a compositon factor of $\Omega M$ with pd $S=d-1$. According to $3.5(1)$, we have pd $S=2 a(S)-1$. Therefore $d-1=\operatorname{pd} S=2 a(S)-1 \leq 2 a(A)-1$, thus $d \leq 2 a(A)$.

3.11. Historical Remarks. Some important properties of Nakayama algebras which we use in order to apply the general theory are collected in 3.5. One should be aware that both assertions of 3.5 can be found (at least implicitly) in the literature. The odd modules have been studied quite carefully by Madsen. For the calculation of pd $I S$, where $S$ is a $\psi$-cyclic simple module, we should also refer to Shen, see Lemma 3.5 (2) of [Sh3].

\section{The homological permutation.}

Let $A$ be a Nakayama algebra and $\mathcal{E}$ the set of (isomorphism classes of) simple modules. We are going to construct a permutation $h: \mathcal{E} \rightarrow \mathcal{E}$ related to projective resolutions and injective coresolutions. The essential step in the construction is the following Theorem.

4.1. Theorem. Let $A$ be a Nakayama algebra. Let $S, T$ be simple modules and $z \in \mathbb{N}_{0}$.

(a) If $z$ is even, $\Omega^{z} I T=P S$, and $\operatorname{pd} T \geq z$, then $\Sigma^{z} P S=I T$ and id $S \geq z$.

(b) If $z$ is odd, $\Omega^{z} T=P S$, and $\operatorname{pd} I T \geq z$, then $\Sigma^{z} S=I T$ and id $P S \geq z$.

The proof will be given in section 5 (first, in case $A$ is a linear Nakayama algebra, see 5.1; then, using covering theory, also for $A$ being a cyclic Nakayama algebra, see 5.4).

4.2. Reformulations. Recall that if $S$ is a simple module, then $e(S)=\min \{\operatorname{pd} S, \operatorname{pd} I S\}$. Dually, we have defined $e^{*}(S)=\min \{\operatorname{id} S$, id $P S\}$. For any natural number $z$, let $\mathcal{E}(z)$ be 
the set of simple modules $S$ with $e(S)=z$, and $\mathcal{E}^{*}(z)$ the set of simple modules $S$ with $e^{*}(S)=z$.

If $S$ is a simple module, let

$$
\begin{gathered}
N S=\left\{\begin{array}{cc}
S & \text { if } e(S) \text { is odd } \\
I S & \text { if } e(S) \text { is even. }
\end{array}\right. \\
N^{*} S=\left\{\begin{array}{cc}
S & \text { if } e^{*}(S) \text { is odd } \\
P S & \text { if } e^{*}(S) \text { is even }
\end{array}\right.
\end{gathered}
$$

Also, let

$$
h(S)=\operatorname{top} \Omega^{e(S)} N S, \quad \text { and } \quad h^{*}(S)=\operatorname{soc} \Sigma^{e^{*}(S)} N^{*} S .
$$

Corollary. The map $h$ is a permutation of $\mathcal{E}$ with inverse $h^{*}$. If $S$ is a simple module, then

$$
e^{*}(h(S))=e(S)
$$

We call $h=h_{A}$ the homological permutation of $\mathcal{E}$.

Note that for $A$ being self-injective, we have $h(S)=\operatorname{top} I S$ and $h^{*}(S)=\operatorname{soc} P S$, for any simple module $S$. Thus, in this case, $h$ is the Nakayama permutation of $A$, as considered by Nakayama himself. (Recall that Nakayama [N, Lemma 2], has characterized the self-injective algebras $B$ by the existence of a permutation $h$ of the set of simple modules such that $\operatorname{soc} P(h(S))=S$, or, equivalently, that $h(S)=$ top $I S$. This permutation $h$, but sometimes also its inverse $h^{*}$, is usually called the Nakayama permutation of $B$.)

We can reformulate the Corollary as follows: Let $z$ be a natural number. If $S$ belongs to $\mathcal{E}(z)$, then $h(S)$ belongs to $\mathcal{E}^{*}(z)$, and $h$ yields a bijection $h_{z}: \mathcal{E}(z) \rightarrow \mathcal{E}^{*}(z)$ with inverse given by $h^{*}$. In particular, it follows that the sets $\mathcal{E}(z)$ and $\mathcal{E}^{*}(z)$ have the same cardinality.

Proof. 4.1 asserts that $h$ maps $\mathcal{E}(z)$ into $\mathcal{E}^{*}(z)$. By duality, $h^{*}$ maps $\mathcal{E}^{*}(z)$ into $\mathcal{E}(z)$. We refer again to 4.1 in order to see that $h^{*} h(S)=S$ and $h h^{*}(S)=S$ for all $S \in \mathcal{E}$.

4.3. The ties. The permutation $h$ is, of course, determined by the set of pairs $(T, h(T))$ with $T \in \mathcal{E}$. For such a pair $(T, S)=(T, h(T))$, the number $e(T)=e^{*}(S)$ plays an important role. Let us stress that the pairs $(T, S)=(T, h(T))$ come equipped with addditional information, the corresponding ties: If $e(T)=0$, thus also $e^{*}(S)=0$, there is given an indecomposable module which is both projective and injective, namely $I T=P S$. If for $e(T)>0$, thus $e^{*}(S)>0$, there are given two long exact sequences. Namely, if $e(T)=2 t \geq 2$ is even, there is given a minimal projective resolution of $I T$ and a minimal injective coresolution of $P S$ (both are long exact sequences starting with $P S$ and ending with $I T$ ), and we use, in addition, the inclusion map $T \rightarrow I T$ and the projection $P S \rightarrow S$ :

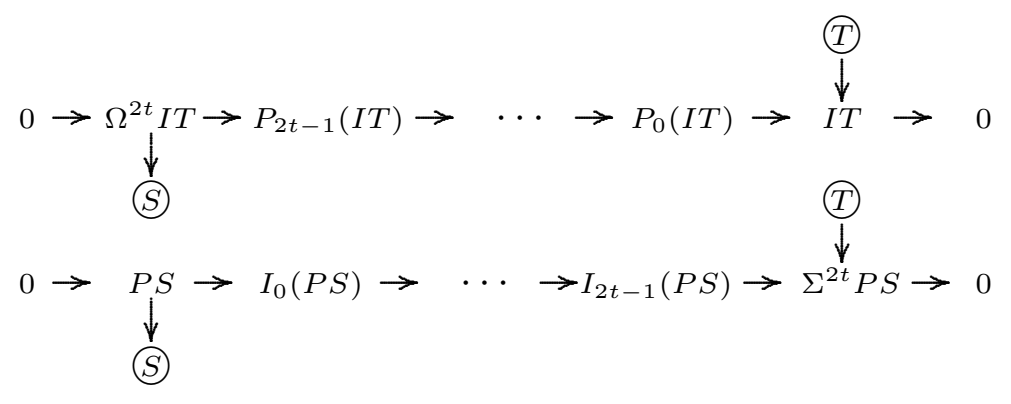


If $e(T)$ is odd, there is given a minimal projective resolution of $T$ (thus a long exact sequence starting with $P S$ and ending with $T$ ), as well as a minimal injective coresolution of $S$ (thus a long exact sequence starting with $S$ and ending with $I T$ ), and we use, in addition, the projection $P S \rightarrow S$ and the inclusion map $T \rightarrow I T$.

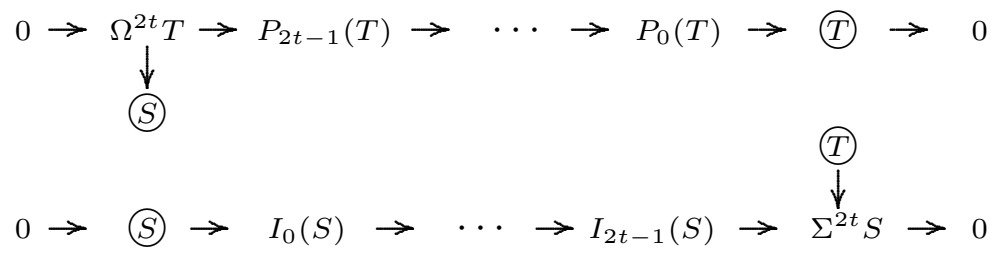

Alternatively, we can say that for any pair $(T, h(T))$, there are given specified walks in the Auslander-Reiten quiver, starting with $T$ and ending in $h(T)$ : For $e(T)=0$, there is given the composition of the sectional path $T \rightarrow I T$ with the sectional path $I T=P S \rightarrow S$. For $e(T)>0$, there is given the walk from $T$ to $S=h(T)$ via the long exact sequences which we have mentioned. Some examples are outlined in 4.5 and 4.6. A more detailled discussion of these ties for small values $z=e(T)$ will be found in 4.7 .

Remark. Let us stress the following: Let $T$ be a simple module. If $z=e(T)$ is odd, then clearly $\operatorname{Ext}^{z}(T, h(T)) \neq 0\left(\right.$ since $h(T)=\operatorname{top} \Omega^{z} T$ and $\operatorname{Ext}^{z}\left(T, \operatorname{top} \Omega^{z} T\right)=$ $\left.\operatorname{Ext}^{z}\left(T, \Omega^{z} T\right)\right)$. However, for $z=e(T)$ even, we may have $\operatorname{Ext}^{z}(T, h(T))=0$. Here is a typical example with $z=2$ : Take for $T$ the simple module with index 4 , thus $h(T)$ is the simple module with index 2 and clearly $\operatorname{Ext}^{2}(T, h(T))=0$.

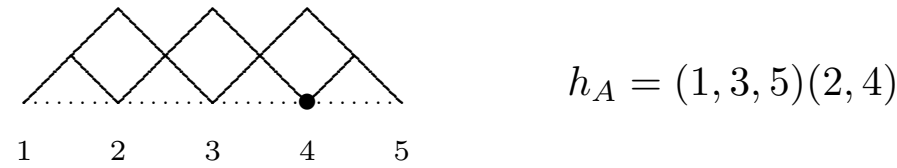

4.4. Combinatorial description. Let us repeat the steps of the algorithm which yields $h_{A}$ for a given Nakayama algebra $A$. Let $\Gamma$ be the Auslander-Reiten quiver of $A$. The south-east arrows of $\Gamma$ correspond to irreducible maps which are epimorphisms, and the north-east arrows correspond to irreducible maps which are monomorphisms.

(1) Given a vertex $x$, the top top $x$ of $x$ is the end of the longest south-east path starting in $x$; the projecive cover $P x$ of $x$ is the start of the longest south-east path $p_{x}$ ending in $x$; the injective envelope $I x$ of $x$ is the end of the longest north-east path starting in $x$. If $x$ is not a projective vertex, the syzygy module $\Omega x$ is the kernel of $p_{x}$ (thus there is a north-east path from $\Omega x$ to $P x$ of length at least 1).

(2) Using inductively $P$ and $\Omega$, we can define the function pd: $\Gamma_{0} \rightarrow \mathbb{N}_{0} \cup\{\infty\}$. For any simple module $S$, define $e(S)=\min \{\operatorname{pd} S, \operatorname{pd} I S\}$.

(3) Now, for any simple module $S$, let $N S=S$ in case $e(S)$ is odd, and $N S=I S$ in case $e(S)$ is even. Finally let $h_{A}(S)=\operatorname{top} \Omega^{e(S)} N S$.

Once we know the values $e(S)$, for $S$ simple, there is an alternative description of $h_{A}$, using the Auslander-Reiten translation $\tau$ as well as the functions $\phi=\operatorname{top} I(-)$ and $\gamma=\tau \operatorname{soc} P(-)$ (but note that it is our convention to apply $\tau$ only to indecomposable non-projective modules, thus $\tau$ and $\gamma$ are not always defined). 
Proposition. If $S$ is a simple module with $e(S)=2 t$, then $h(S)=\gamma^{t} \phi(S)$. If $S$ is a simple module with $e(S)=2 t+1$, then $h(S)=\gamma^{t} \tau(S)$.

Proof. If pd $I S=0$, we have $e(S)=0$ and $h(S)=\operatorname{top} I S=\phi S=h_{0}(S)$. If pd $I S \geq 1$, then also pd $S \geq 1$ and top $\Omega S=\operatorname{top} \Omega I S=\tau S$. First, let $e(S)=2 t$. Then $h(S)=$ top $\Omega^{z} I S=\gamma_{t} \operatorname{top} I S=\gamma^{t} \phi S$. Second, let $e(S)=2 t+1$. Then $h(S)=\operatorname{top} \Omega^{2 t} \Omega S=$ top $\Omega^{2 t} \tau S=\gamma^{t} \tau S$.

We write $h_{z}=h \mid \mathcal{E}(z)$. Thus, the Proposition asserts that for all $t \geq 0$,

$$
h_{2 t}=\gamma^{t} \phi \mid \mathcal{E}(2 t), \quad \text { and } \quad h_{2 t+1}=\gamma^{t} \tau \mid \mathcal{E}(2 t+1) .
$$

Remark. Let us assume that $T$ is simple and that $z=\operatorname{pd} T=\operatorname{pd} I T$. If $z$ is odd, then $\Omega^{z} T=\Omega^{z} I T$, whereas for $z$ even, we many have $\Omega^{z} T \neq \Omega^{z} I T$. Namely, if $z=2 t+1$, then

$$
\text { top } \Omega^{z} T=\gamma^{t} \text { top } \Omega T \text { and top } \Omega^{z} I T=\gamma^{t} \text { top } \Omega I T \text {, }
$$

and top $\Omega T=\tau T=\operatorname{top} \Omega I T$. On the other hand, as in Remark 4.3, we look at the example

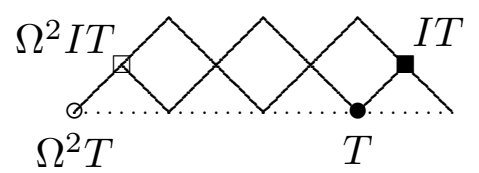

Note that for $\operatorname{pd} T=\operatorname{pd} I T$ odd, a minimal projective resolution of $I T$ as well as a minimal injective coresolution of $P(h(T))$ provide additional interesting ties between $T$ and $h(T)$.

4.5. Linear Nakayama algebras. Some examples and remarks. We want to provide some examples which illustrate the homological permutation and the corresponding ties, and we will add some remarks.

We start with the following example of a linear Nakayama algebra $A$. The simple modules are labeled $1,2, \ldots, 5$

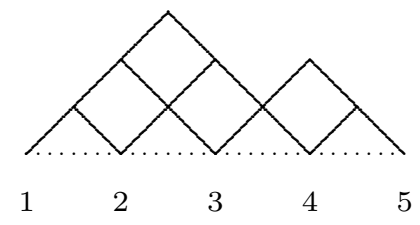

Let us indicate ties for the simple modules $T$. Here, $T$ is always marked by a bullet; for $T=2$ and $T=4$, we draw just one of the walks in the Auslander-Reiten quiver (the one which uses a minimal projective resolution of $T$ ).

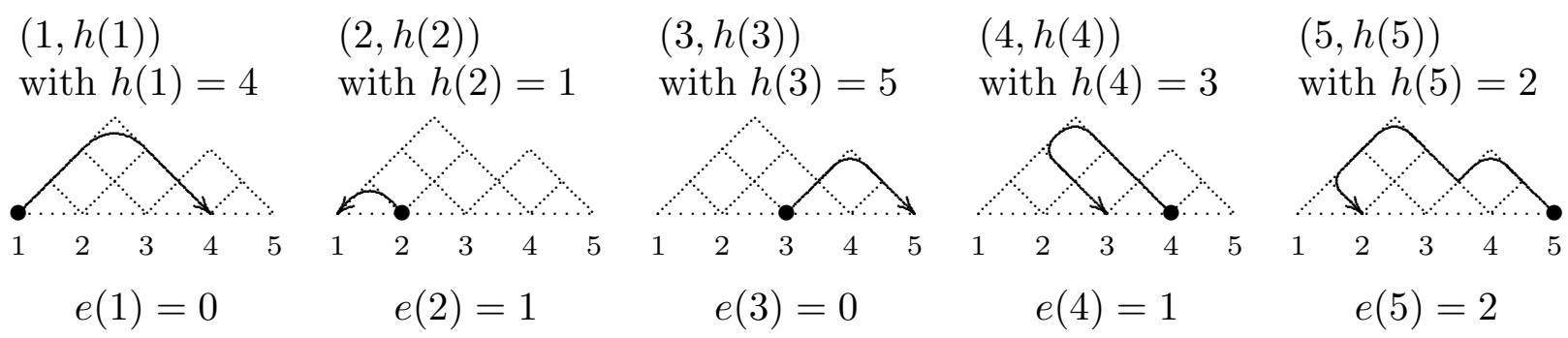


The permutation $h$ is, in cycle notation, the permutation (14352).

Here are the permutations $h_{A}$ which occur for a linear Nakayama algebra $A$ with 4 simple modules.

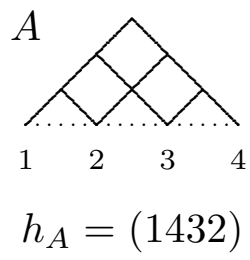

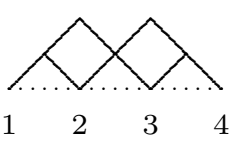

$(1324)$

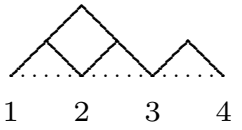

$(1342)$

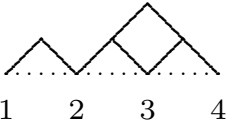

$(1243)$

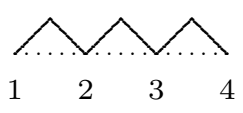

$(1234)$

Remarks. We start with some basic observation concerning linear Nakayama algebras $A$. We assume that $\mathcal{E}=\{1,2, \cdots, n\}$ with $\tau x=x-1$ for $2 \leq x \leq n$.

(1) If $e(x)=0$, then $h_{A}(x)>x$. If $e(x)>0$, then $h_{A}(x)<x$.

(2) As a consequence, $h_{A}$ is a fixed-point-free permutation of $\{1,2, \cdots, n\}$.

(3) Already the pairs $\left(x, h_{A}(x)\right)$ with $h(x)>x$ determine the algebra A: namely, they determine the peaks, thus the Kupisch series of $A$ (a peak of $A$ is by definition an indecomposable module which is projective and injective). In particular, $A$ is determined by $h_{A}$.

(4) Note that the linear Nakayama algebras with $n \geq 1$ simple modules correspond bijectively to the Dyck paths of length $2(n-1)$. Namely, the roof of $A$ is (say after a rotation by 225 degrees) a Dyck path $D_{A}$ :
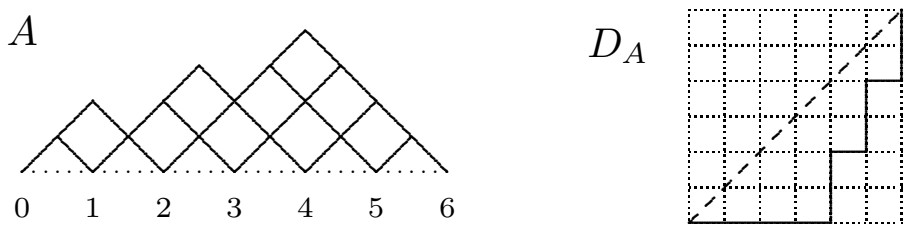

$$
h_{A}=(0265)(143)
$$

The number of Dyck paths of length $2 m$ (thus the number of linear Nakayama algebras with $m+1$ simple modules) is the Catalan number $C_{m}=\frac{1}{m+1}\left(\begin{array}{c}2 m \\ m\end{array}\right)$ : we are in the center of Catalan combinatorics. There are several well-known procedures to attach to Dyck paths permutations, but usually one attaches to a Dyck path of length $2 m$ a permutation of $m$ objects, not of $m+1$ objects, as we do it here. We are not aware that the correspondence $D_{A} \mapsto h_{A}$ has yet been considered in combinatorics (but the number of papers on Catalan combinatorics seems to be too large to be overlooked) - if it has, our discussion may add at least a homological interpretation.

(5) M. Rubey has added the homological permutation for linear Nakayama algebras to the database FindStat, see $[\mathrm{RS}]$.

4.6. Cyclic Nakayama algebras. Some examples (and remarks). Let $A$ be the following cyclic Nakayama algebra with simple modules with labels 1, 2,3,4

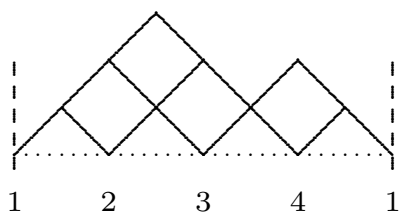


Here are ties for the simple modules $S$

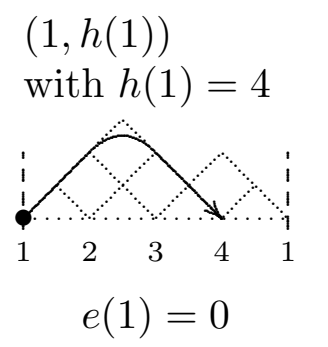

$$
(2, h(2))
$$

with $h(2)=2$

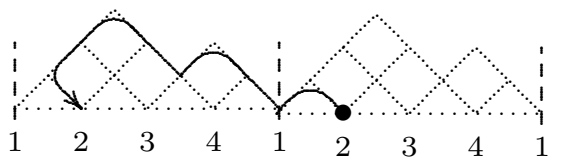

$e(2)=3$
$(3, h(3))$

with $h(3)=1$

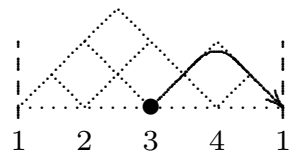

$e(3)=0$
$(4, h(4))$ with $h(4)=3$

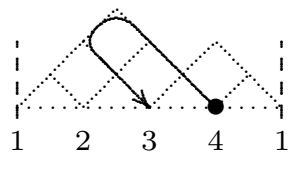

$e(4)=1$

The permutation $h$ is, in cycle notation, the permutation (143)(2).

Remarks. (1) As we see, the permutation $h_{A}$ may have fixed points.

(2) The permutation $h_{A}$ does not determine the algebra $A$. There are many cyclic Nakayama algebras say with two simple modules, but of course just two permuations of $\{1,2\}$. For example, the algebras
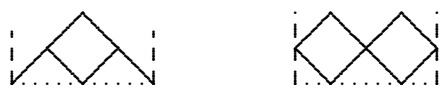

both have as homological permutation the identity permutation $h_{A}=(1)(2)$.

4.7. The sets $\mathcal{E}(z)$. If $z$ is a natural number, then $\mathcal{E}(z)$ is the set of simple modules $S$ with $e(S)=z$ (thus, for any natural number $t$, the set $\mathcal{E}(2 t)$ consists of all simple modules $S$ with $2 t=\operatorname{pd} I T \leq \operatorname{pd} T$, and the set $\mathcal{E}(2 t+1)$ consists of all simple modules $S$ with $2 t+1=\operatorname{pd} T \leq \operatorname{pd} I T)$. Of course, the sets $\mathcal{E}(z)$ form a partion of $\mathcal{E}$.

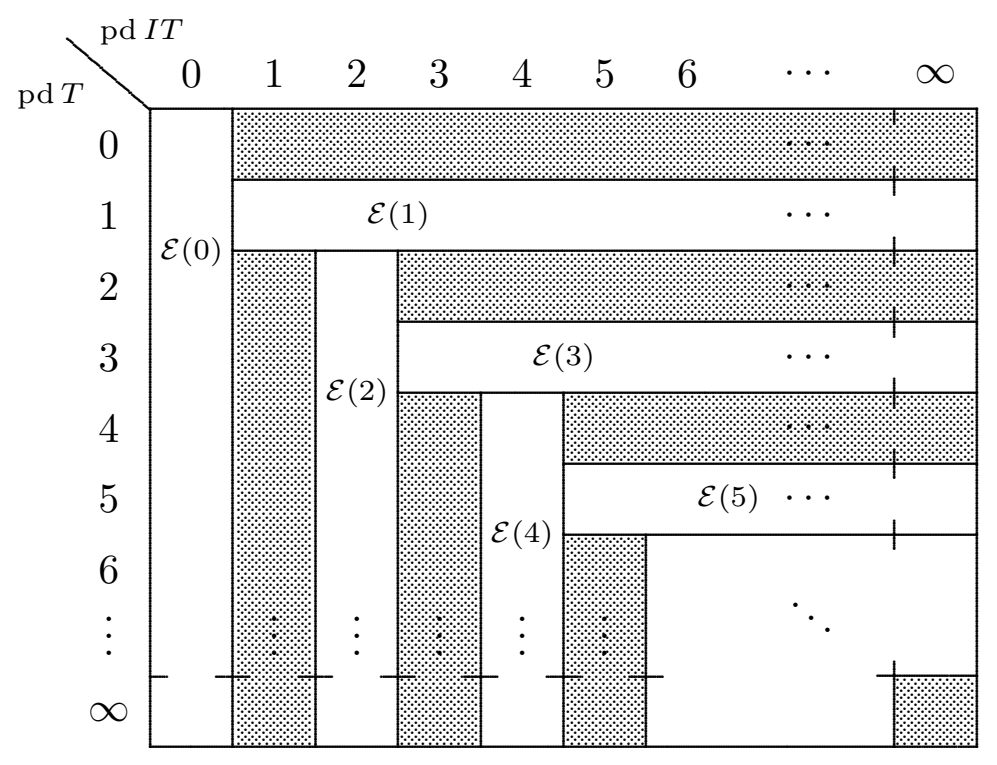

In case $A$ is connected and has infinite global dimension, then, for $T$ simple, $\operatorname{pd} T$ 
cannot be even, pd IT cannot be odd, thus it is sufficient to look at the following display:

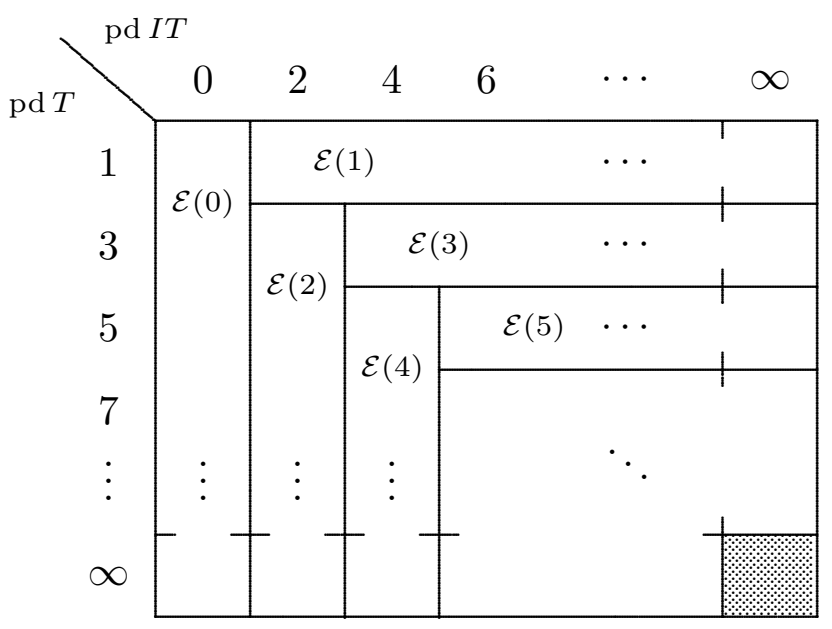

Let us return to the general case where $A$ has arbitrary global dimension. We show: all cases $(p, q)=(\operatorname{pd} S, \operatorname{pd} I S)$ which are not shaded, actually can occur.

Proof.

Of course, the case $(0,0)$ occurs only if $A$ has a connected component with linear quiver (the number of connected components with linear quiver is just the number of simple modules $S$ with pd $S=0)$. On the other hand, the cases $(z, \infty)$ and $(\infty, z)$ can occur only in case the global dimension of $A$ is infinite.

In the cases $(z, \infty)$ and $(\infty, z)$, the examples provided are cyclic Nakayama algebras (of course). If $(p, q) \neq(0,0)$ and $p \neq \infty$, and $q \neq \infty$, we provide translation quivers which are the Auslander-Reiten quivers of a non-cyclic Nakayama algebra, and which also can be part of the Auslander-Reiten quiver of a cyclic Nakayama algebra.

The case $(0,0)$. If $A$ is a Nakayama algebra with a simple projective module $S$, then $\operatorname{pd} S=0, \operatorname{pd} I S=0$.

The case $(t, 0)$ with $t \geq 1$.

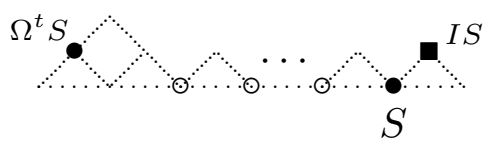

The case $(\infty, 0)$.

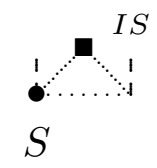

The case $(1,1)$.

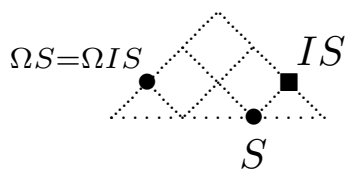


The case $(z, z)$ with $z \geq 2$.

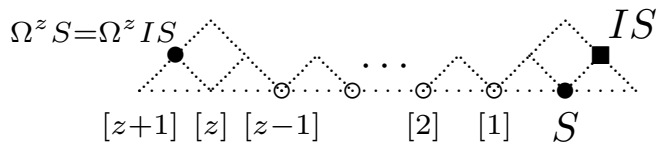

In this example, we have $\Omega^{i} S=\Omega^{i} I S=[i]$, for $1 \leq i \leq z-1$, and $\Omega^{z} S=\Omega^{z} I S=P[z]$.

If $z=\operatorname{pd} S=\operatorname{pd} I S$ is odd, then always $\Omega^{z} S=\Omega^{z} I S$. However, if $z=\operatorname{pd} S=\operatorname{pd} I S$ is even, then we may have that $\Omega^{z} S \neq \Omega^{z} I S$, as the following example shows (with $z=2 t$ ):

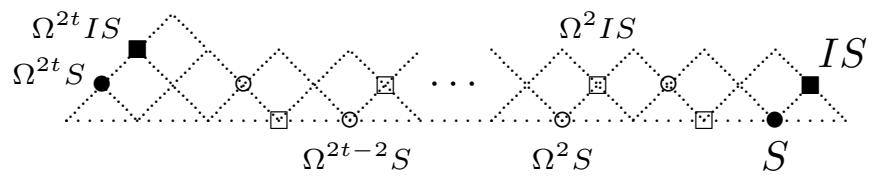

The case $(2 t+u, 2 t)$ with $u \geq 1$ and $t \geq 1$. Let $v=2 t+u$.

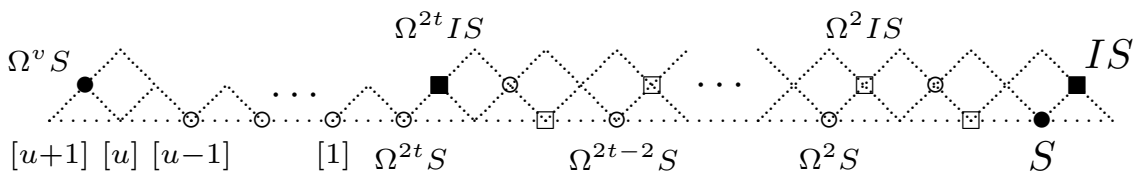

where, on the right, starting with $\Omega^{2 t} S$ and ending with $\tau^{-} S$, there are $3 t+2$ simple modules (thus altogether there are $3 t+u+3$ simple modules). Note that $\Omega^{2 t} S$ is projective, thus pd $S=2 t$. Of course, also $\Omega^{v} S=\Omega^{2 t+u} S=\Omega^{u}\left(\Omega^{2 t} S\right)$ is projective, thus pd $I S=$ $v=2 t+u$.

The case $(\infty, 2 t)$ with $t \geq 1$.

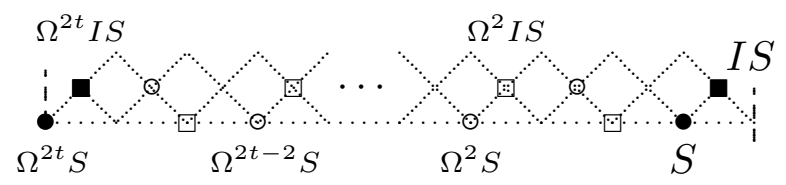

It is easy to check that $\Omega^{2 t} S$ is $\Omega$-periodic. Thus $\operatorname{pd} S=\infty$.

The case $(2 t+1,2 t+2)$ with $t \geq 0$.

$\bmod A^{\prime \prime}$

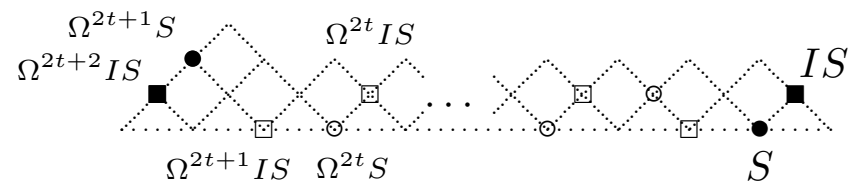

The case $(2 t+1,2 t+2+u)$ with $u \geq 1$ and $t \geq 1$. Let $v=2 t+2+u$.

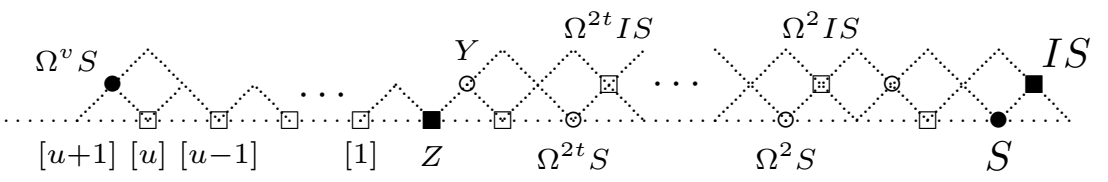


with $Y=\Omega^{2 t+1} S$ and $Z=\Omega^{2 t+2} I S$. On the right, starting with $Z=\Omega^{2 t+2} S$ and ending with $\tau^{-} S$, there are $3 t+5$ simple modules (thus altogether there are $3 t+u+6$ simple modules). Note that $Y=\Omega^{2 t+1} S$ is projective, thus pd $S=2 t+1$. Of course, also $\Omega^{v} S=\Omega^{2 t+2+u} S=\Omega^{u}\left(\Omega^{2 t+2} S\right)=\Omega^{2} Z$ is projective, thus pd $I S=v=2 t+2+u$.

The case $(2 t+1, \infty)$ with $t \geq 0$.

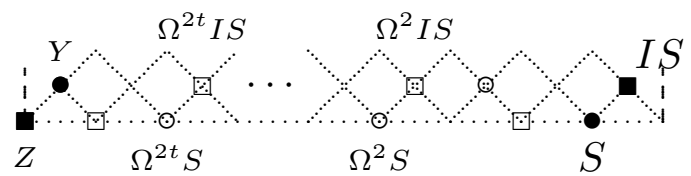

Again, $Y=\Omega^{2 t+1} S$ and $Z=\Omega^{2 t+2} I S$. What we have to verify (but this is easy), is that $Z$ is $\Omega$-periodic.

Remark. Let $A$ be a cyclic Nakayama algebra. Then $a(A)$ has been defined to be the maximum of the numbers $a(S)$, where $S$ is simple and not $\psi$-cyclic.

The set $\mathcal{E}(2 a(A))$ consists of the $\psi$-cyclic simple modules $S$ with $\operatorname{pd} I S=2 a(A)$. Similarly, $\mathcal{E}^{*}(2 a(A))$ consists of the $\gamma$-cyclic simple modules $S$ with id $P S=2 a(A)$.

Proof. Let $a=a(A)$. As we have seen in 3.10, we have fin-pro $A \leq 2 a$. Assume that $S$ is a simple module in $\mathcal{E}(2 a)$. Then $\operatorname{pd} S \geq 2 a$ and $\operatorname{pd} I S=2 a$. Since fin-pro $A \leq 2 a$, it follows from pd $S \geq 2 a$ that $S$ cannot be odd, thus $S$ is $\psi$-cyclic. Conversely, assume that $S$ is $\psi$-cyclic and $\operatorname{pd} I S=2 a$. Then $\operatorname{pd} S$ is even or infinite. But if $\operatorname{pd} S$ is even, then $\operatorname{pd} S \geq \operatorname{pd} I S$, see 3.2 (b). We see that always pd $S \geq 2 a$, therefore $S \in \mathcal{E}(2 a)$.

4.8. Ties for small values of $z$. We are going to look at small values of $z$, and describe the bijection $h_{z}: \mathcal{E}(z) \rightarrow \mathcal{E}^{*}(z)$.

The case $z=0$.

Let $S, T$ be simple modules. Then $T$ belongs to $\mathcal{E}(0)$ iff $T$ is torsionless, iff $I T$ is projective. Also, $S$ belongs to $\mathcal{E}^{*}(0)$ iff $S$ is divisible, iff $P S$ is projective.

The bijection $h_{0}: \mathcal{E}(0) \rightarrow \mathcal{E}^{*}(0)$ sends a simple torsionless module $T$ to top $I T$, the inverse sends a simple divisible module to soc $P S$.

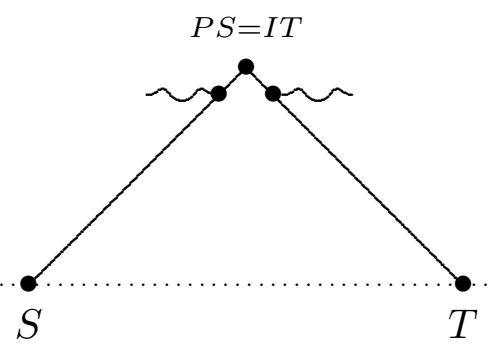

The case $z=1$.

Let $S, T$ be simple modules. Then $T$ belongs to $\mathcal{E}(1)$ iff $T$ is not torsionless, and $\operatorname{pd} T=1$. And $S$ belongs to $\mathcal{E}^{*}(1)$ iff $S$ is not divisible, and id $S=1$. 
The bijection $h_{1}: \mathcal{E}(1) \rightarrow \mathcal{E}^{*}(1)$ is given by the Auslander-Reiten translation $\tau$.

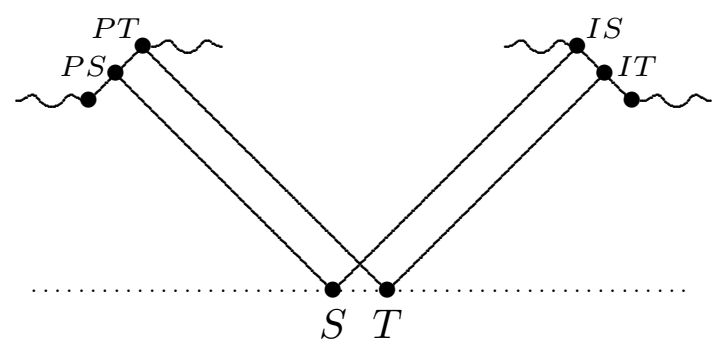

Remark. 1-free algebras. We say that a Nakayama algebra is 1 -free provided $\mathcal{E}(1)$ is empty. Given a Nakayama algebra $A$, we may delete all indecomposable modules $M$ with soc $M$ or top $M$ in $\mathcal{E}(1)$ and obtain the module category of a 1-free algebra $A^{\prime}$ which has similar properties as $A$.

The case $z=2$.

Let $S, T$ be simple modules. Then $T$ belongs to $\mathcal{E}(2)$ iff $\operatorname{pd} I T=2$ and $\operatorname{pd} T \geq 2$. And, $S$ belongs to $\mathcal{E}^{*}(2)$ iff id $P S=2$ and id $S \geq 2$.

The bijection $h_{2}: \mathcal{E}(2) \rightarrow \mathcal{E}^{*}(2)$ sends $T$ to $\gamma \phi(T)=\tau$ soc PIT. (Recall that $\phi(T)=$ top $I T$ and $\gamma\left(T^{\prime}\right)=\tau \operatorname{soc} P\left(T^{\prime}\right)$.)

We have the following diagram.

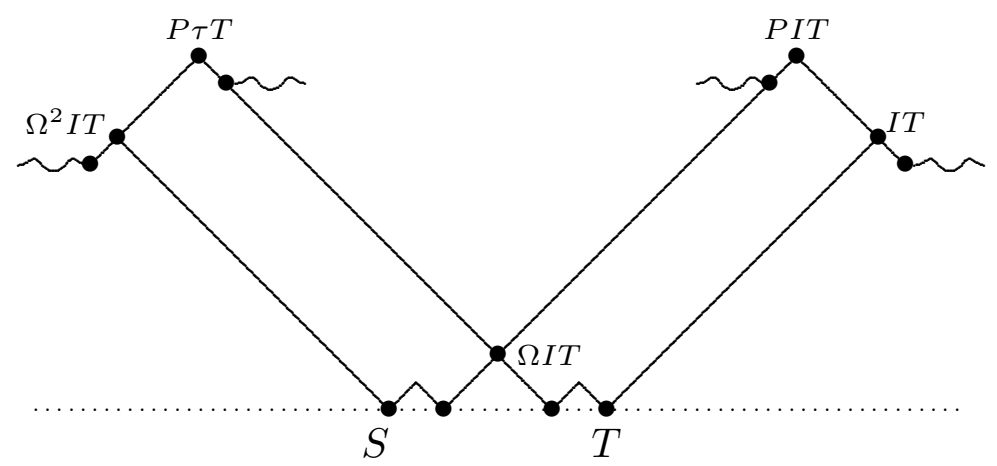

Note that $S=\tau^{a+1} T$, where $a=|\Omega I T|$. The modules $\tau^{i} T$ with $1 \leq i<a$ belong to $\mathcal{E}(1)$. If $A$ is 1-free, then $\Omega I T=\tau T$, so that $a=1$ and therefore $h(T)=S=\tau^{2} T$ and $\tau T$ is both torsionless and divisible.

4.9. Remark. As we have mentioned, $\mathcal{E}(0)$ consists of the torsionless simple modules, thus $|\mathcal{E}(0)|=r$, where $r$ is the number of peaks of $A$. We also have $\left|\bigcup_{z>2} \mathcal{E}(z)\right| \leq r$. Namely, if $S$ is simple and $e(S) \geq 2$, then $\tau S$ is divisible, thus $\tau$ maps $\bigcup_{z \geq 2} \mathcal{E}(z)$ into the set $\mathcal{E}^{*}(0)$. Since $\tau$ (restricted to $\left.\bigcup_{z \geq 2} \mathcal{E}(z)\right)$ is injective, and $\left|\mathcal{E}^{*}(0)\right|=r$, the assertion follows.

4.10. Remark. We have seen in 3.7 (4) and (5) that for calculating fin-pro $A$, we may look at the functions $e$ or $f$. Whereas $f$ is easier to handle, the importance of $e$ lies in 
the symmetry assertion formulated in Corollary 4.2. There is no corresponding assertion for $f$, as we want to show now. Thus, let $f^{*}(S)=0$ in case id $S$ is odd and id $P S$ is even, $f^{*}(S)=$ id $S$ in case id $P S$ is not even, and $f^{*}(S)=\operatorname{id} P S$ in case id $S$ is not odd. In general, there is no permutation $\pi$ of the simple modules with $f^{*}(\pi S)=f(S)$ for all simple modules $S$, see the following example.

\section{Example.}

$\bmod A$ with pd

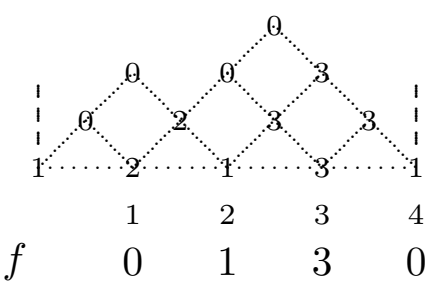

$\bmod A$ with id

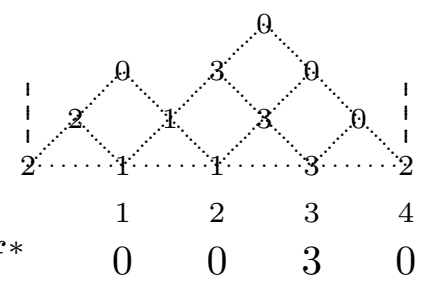

On the left, we have equipped the Auslander-Reiten quiver with the values $\operatorname{pd} M$; on the right, with the values id $M$. We label the simple modules by 1 up to 4 as shown below the Auslander-Reiten quivers and provide the values $f(S)$ or $f^{*}(S)$, respectively, below the label of $S$. We see that there is a simple module $S$, namely the one with index 2 , such that $f(S)=1$. But there is no simple module $S$ with $f^{*}(S)=1$.

One may also ask, whether there is a permutation $\pi$ of $\mathcal{E}$ such that $\operatorname{des} \pi(S)=\operatorname{del} S$ for any simple module $S$. Of course, this is true in case del $S=e(S)$ for all simple modules $S$ (but, as we have mentioned, this is not known, see 3.8).

\section{Proof of Theorem 4.1.}

Let $A$ be a connected Nakayama algebra. First, we consider the case that $A$ is linear. In case $A$ is cyclic, we will use covering theory in order to reduce it to the linear case.

5.1. The case of $A$ being a linear Nakayama algebra. Let $A$ be a linear Nakayama algebra. The proof of the assertions (a) and (b) will be quite easy, as soon as we have introduced suitable labels for the modules involved.

Dealing with a linear Nakayama algebra $A$, we identify the set of simple modules with a finite interval of $\mathbb{Z}$, say with $[\alpha, \omega] \subset \mathbb{Z}$, with $\alpha \leq \omega$, such that the Auslander-Reiten translation $\tau$ is given by $\tau x=x-1$. By abuse of notation, we also write $\tau(\omega+1)=\omega$. In this way, the indecomposable modules can be written in the form $[x, y[$, where $\alpha \leq x<$ $y \leq \omega+1$. We have $\operatorname{soc}[x, y[=x$ and top $[x, y[=\tau y$. This convention has the following advantage: if $[x, y[$ is non-projective, then $\Omega[x, y[=[\operatorname{soc} P \tau y, x[$. Similarly, if $[x, y$ [ is noninjective, then $\Sigma\left[x, y\left[=\left[y, \tau^{-}\right.\right.\right.$top $I x[$. This fact should be considered as the start of the general setting which we will use.

(1) For a pair $V \subseteq W$ of indecomposable non-projective modules with the same socle, let $P_{0}$ and $Q_{0}$ be the projective covers and $\Omega V, \Omega W$ the first syzygy modules, of $V$ and $W$, respectively. Let $\operatorname{soc} V=\operatorname{soc} W=u_{0}$ and top $V=\tau v_{0}$, top $W=\tau w_{0}$, thus $V=\left[u_{0}, v_{0}\left[\right.\right.$ and $W=\left[u_{0}, w_{0}\left[\right.\right.$. Let $P_{0}=\left[v_{1}, v_{0}\left[\right.\right.$ and $Q_{0}=\left[w_{1}, w_{0}\left[\right.\right.$, then $v_{1} \leq w_{1}<u_{0}$ 
(the strict inequality is due to the fact that we assume that $W$ is not projective), and $\Omega V=\left[v_{1}, u_{0}\left[, \Omega W=\left[w_{1}, u_{0}[\right.\right.\right.$.

For an indecomposable non-projective module $V^{\prime}$ with a non-zero factor module $W^{\prime}$, let $B_{1}$ be a projective cover of $V^{\prime}$ and $\Omega V^{\prime} \subseteq \Omega W^{\prime} \subset B_{1}$ the syzygy modules of $V^{\prime}$ and $W^{\prime}$ respectively. Let $\operatorname{soc} V^{\prime}=v_{1}$, soc $W^{\prime}=w_{1}$ and top $V^{\prime}=\operatorname{top} W^{\prime}=\tau u_{0}$. The projective cover of $V^{\prime}$ ad $W^{\prime}$ is $B^{\prime}=\left[u_{1}, u_{0}\right.$ [ for some $u_{1}<v_{1}$ and we have $\Omega V^{\prime}=\left[u_{0}, v_{1}\right.$ [ and $\Omega W^{\prime}=\left[u_{1}, w_{1}[\right.$.

Thus, there are the following two pictures: on the right, we start with the pair $V \subseteq W$, on the left, we start with $V^{\prime}$ and its factor module $W^{\prime}$.
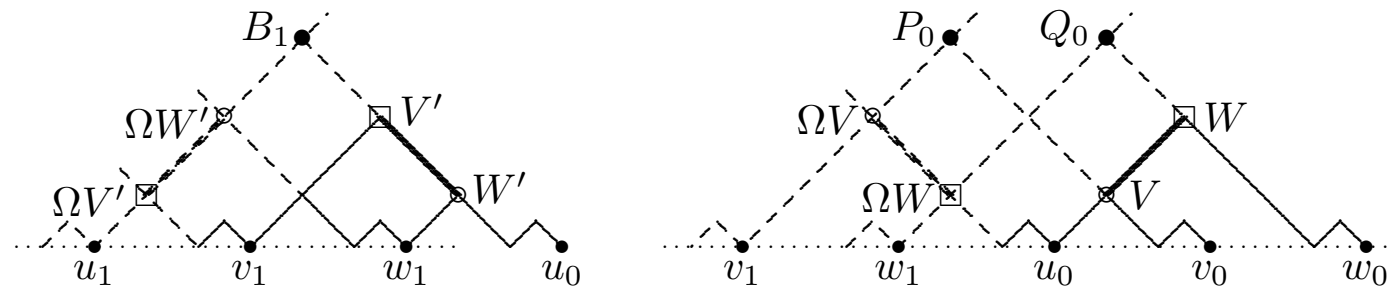

(2) We use successively such labels in order to obtain the first $z$ terms of minimal projective resolutions (and the corresponding syzygy modules) of say modules $V \subseteq W$ with socle $T$, where pd $V \geq z, \operatorname{pd} W \geq z$ (the case, we are interested in, is $V=T, W=I T$, but first we look at the general case in order to highlight the pattern).

We assume that $V \subseteq W$ are modules with socle $T$, such that $\operatorname{pd} V \geq z$ and $\operatorname{pd} W=z$. Let

$$
u_{0}=T, \quad v_{0}=\tau^{-} \operatorname{top} V, \quad w_{0}=\tau^{-} \operatorname{top} W .
$$

Also, let $S=\operatorname{top} \Omega^{z} W$.

Let us denote by $Q_{j}$ and $P_{j}$ with $0 \leq j \leq z$ the first projective modules which occur in a minimal projective resolution of $V$ and $W$, respectively. For $j$ odd, we have $P_{j}=Q_{j}$, thus let $B_{j}=P_{j}=Q_{j}$ for $j$ odd.

In case $z$ is odd, say $z=2 t+1$, the minimal projective resolutions of $V$ and $W$ are of the form

$$
\begin{gathered}
\cdots \rightarrow B_{2 t+1} \rightarrow P_{2 t} \rightarrow \cdots \rightarrow B_{3} \rightarrow P_{2} \rightarrow B_{1} \rightarrow P_{0} \rightarrow V \rightarrow 0 \\
0 \rightarrow B_{2 t+1} \rightarrow Q_{2 t} \rightarrow \cdots \rightarrow B_{3} \rightarrow Q_{2} \rightarrow B_{1} \rightarrow Q_{0} \rightarrow W \rightarrow 0
\end{gathered}
$$

In case $z$ is even, say $z=2 t$, the minimal projective resolutions of $V$ and $W$ look as follows

$$
\begin{gathered}
\cdots \rightarrow P_{2 t} \rightarrow B_{2 t-1} \rightarrow \cdots \rightarrow B_{3} \rightarrow P_{2} \rightarrow B_{1} \rightarrow P_{0} \rightarrow V \rightarrow 0 \\
0 \rightarrow Q_{2 t} \rightarrow B_{2 t-1} \rightarrow \cdots \rightarrow B_{3} \rightarrow Q_{2} \rightarrow B_{1} \rightarrow Q_{0} \rightarrow W \rightarrow 0
\end{gathered}
$$

We define for $i \geq 1$

$$
u_{i}=\operatorname{soc} B_{2 i-1}, \quad v_{i}=\operatorname{soc} P_{2 i-2}, \quad w_{i}=\operatorname{soc} Q_{2 i-2} .
$$


Thus, we obtain for $z=2 t+1$ a sequence of integers

$$
v_{t+1} \leq w_{t+1}<u_{t}<v_{t} \leq w_{t}<\cdots<u_{1}<v_{1} \leq w_{1}<u_{0}<v_{0} \leq w_{0} .
$$

and for $z=2 t$ the subsequence

$$
u_{t}<v_{t} \leq w_{t}<\cdots<u_{1}<v_{1} \leq w_{1}<u_{0}<v_{0} \leq w_{0}
$$

Always, we have

$$
B_{2 i+1}=\left[u_{2 i+1}, u_{2 i}\left[, \quad P_{2 i}=\left[v_{2 i+1}, v_{2 i}\left[, \quad Q_{2 i}=\left[w_{2 i+1}, w_{2 i}[,\right.\right.\right.\right.\right.
$$

and

$$
\begin{aligned}
\Omega^{2 i} V & =\left[u_{2 i}, v_{2 i}\left[, \quad \Omega^{2 i+1} V=\left[v_{2 i+1}, u_{2 i}[,\right.\right.\right. \\
\Omega^{2 i} W & =\left[u_{2 i}, w_{2 i}\left[, \quad \Omega^{2 i+1} W=\left[v_{2 i+1}, u_{2 i}[.\right.\right.\right.
\end{aligned}
$$

This is the labelling of the relevant modules which we are going to use.

For a better vision, let us exhibit the special cases $z=6$ and $z=7$. Here, $t=3$. First, we show the case $z=2 t=6$, and second the case $z=2 t+1=7$.

$$
t=3 \quad z=2 t=6
$$

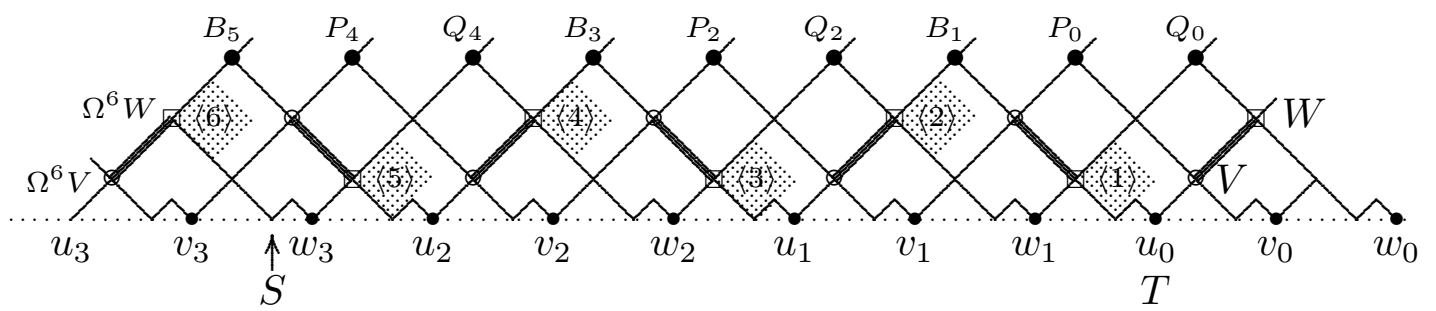

$t=3 \quad z=2 t+1=7$

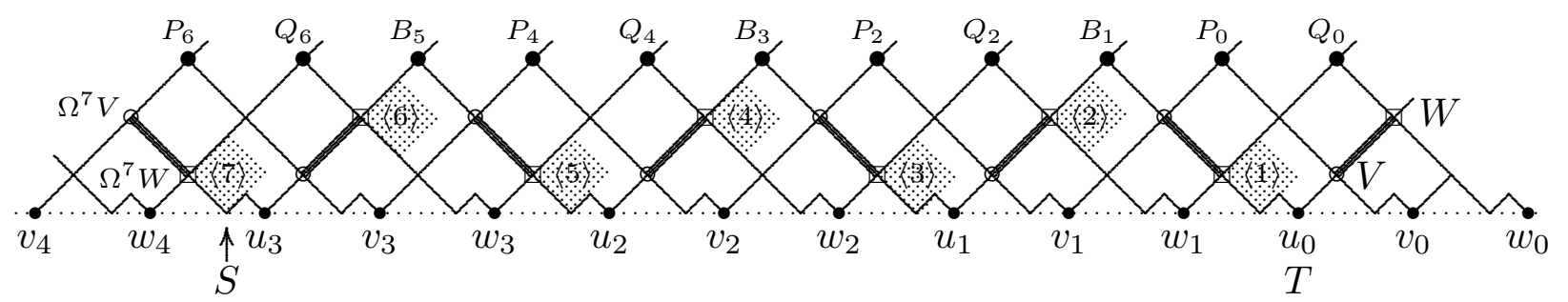

The shaded areas with labels of the form $\langle i\rangle$ will be defined in (3):

(3) We define $\langle j\rangle$ for $j \geq 1$ as follows: $\langle 2 i\rangle$ is the set of modules $\left[x, y\right.$ [ with $u_{i} \leq x<v_{i}$ and $w_{i} \leq y<u_{i-1}$ whereas $\langle 2 i-1\rangle$ is the set of modules $\left[x, y\right.$ [ with $w_{i} \leq x<u_{i-1}$ and $u_{i-1} \leq y<v_{i-1}$. The following assertion is essential:

If $M$ belongs to $\langle j\rangle$ with $j \geq 2$, then $\Sigma M \in\langle j-1\rangle$.

Proof. First, let $j=2 i$ be even (thus $i \geq 1$ ). Let $M=\left[x, y\left[\in\langle 2 i\rangle\right.\right.$, thus $u_{i} \leq x<v_{i}$ and $w_{i} \leq y<u_{i-1}$. Then $I M=I x$. Since $B_{2 i-1}=\left[u_{i}, u_{i-1}\left[\right.\right.$ has $x$ and $\tau u_{i-1}$ as 
composition factors, we see that $\tau u_{i-1}$ is a composition factor of $I x$, thus $\tau u_{i-1} \leq \operatorname{top} I M$. On the other hand, $P_{2 i-2}=\left[v_{i}, v_{i-1}\left[\right.\right.$, thus top $P_{2 i-2}=\tau v_{i-1}$. There is no indecomposable module with both $x$ and $\tau v_{i-1}$ as compostion factors, since such a module would have $P_{2 i-2}$ as a proper factor module. It follows that $\tau v_{i-1}$ is not a composition factor of $I M$. Altogether we see that $\tau u_{i-1} \leq \operatorname{top} I M<\tau v_{i-1}$, thus $u_{i-1} \leq \tau^{-} \operatorname{top} I M<v_{i-1}$. Therefore $\Sigma M=\left[y, \tau^{-}\right.$top $I M[$ belongs to $\langle 2 i-1\rangle$.

Next, let $j=2 i-1$ be odd (thus $j \geq 2$ ). Let $M=\left[x, y\left[\in\langle 2 i-1\rangle\right.\right.$, thus $w_{i} \leq x<u_{i-1}$ and $u_{i-1} \leq y<v_{i-1}$. Again, $I M=I x$. The existence of $Q_{2 i-2}=\left[w_{i}, w_{i-1}[\right.$ shows that $\tau w_{i-1}$ is a composition factor of $I M$, thus $\tau w_{i-1} \leq \operatorname{top} I M$. The fact that $B_{2 i-3}=$ $\left[u_{i-1}, u_{i-2}\left[\right.\right.$ is projective implies that $\operatorname{top} I M<\tau u_{i-2}$. Altogether we see that $\tau w_{i-1} \leq$ top $I M<\tau u_{i-2}$, thus $w_{i-1} \leq \tau^{-} \operatorname{top} I M<u_{i-2}$. Therefore $\Sigma M=\left[y, \tau^{-}\right.$top $I M$ [ belongs to $\langle 2 i-1\rangle$.
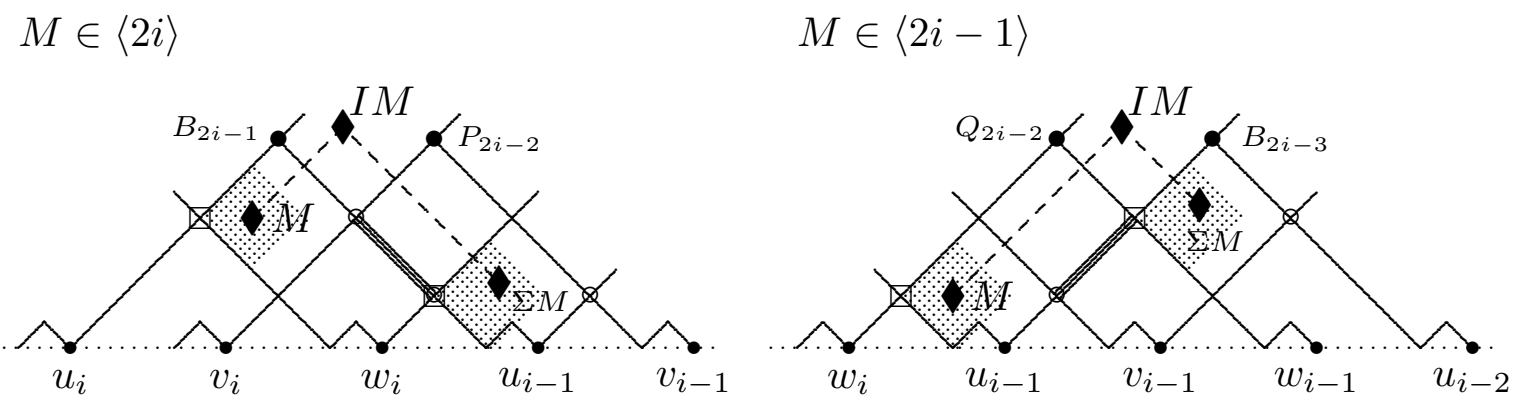

(4) If $V=T$, then $\langle 1\rangle$ is the set of modules $\left[x, u_{0}\left[\right.\right.$ with $w_{1} \leq x<u_{0}$. If $W=I T$, and $w_{1} \leq x<u_{0}$, then $\Sigma\left[x, u_{0}\right]=I T$.

Proof. First, let $V=T$. Now $\langle 1\rangle$ is the set of modules $[x, y]$ with $w_{1} \leq x<u_{0}$ and $u_{0} \leq y<v_{0}$. Let us assume that $V=T$. Then $V=\left[u_{0}, v_{0}\right.$ [ is simple, thus $\tau v_{0}=u_{0}$. The condition $u_{0} \leq y<v_{0}$ is only satisfied for $y=v_{0}$. This is the first assertion.

Next, Let $M=\left[x, u_{0}\left[\right.\right.$ with $w_{1} \leq x<u_{0}$. Then $I M=I x$. The module $Q_{0}=\left[w_{1}, w_{0}[\right.$ has $\left[x, w_{0}\left[\right.\right.$ as a factor module, thus this is a submodule of $I x$. But $W=\left[u_{0}, w_{0}[\right.$ is a factor module of $\left[x, w_{0}\left[\right.\right.$. We assume here that $W=I T=I u_{0}$. Since the injective module $W$ is a factor module of $\left[x, w_{0}\left[\right.\right.$, also $\left[x, w_{0}\right.$ [ has to be injective, thus equal to $I x$. It follows from $I x=\left[x, w_{0}\left[\right.\right.$, that $\Sigma\left[x, u_{0}\left[=\left[u_{0}, w_{0}[=W=I T\right.\right.\right.$.

Remark. If $V=T$ and $W=I T$, let us define $\langle 0\rangle=\{I T\}$. Then we have: If $M \in\langle 1\rangle$, then $\Sigma M \in\langle 0\rangle$, thus If $M$ belongs to $\langle j\rangle$ with $j \geq 1$, then $\Sigma M \in\langle j-1\rangle$.

In the case $V=T, W=I T$ (and this is the case we are interested in), the pictures which we have used are are not quite satisfactory. Here is a better one of the relevant part:

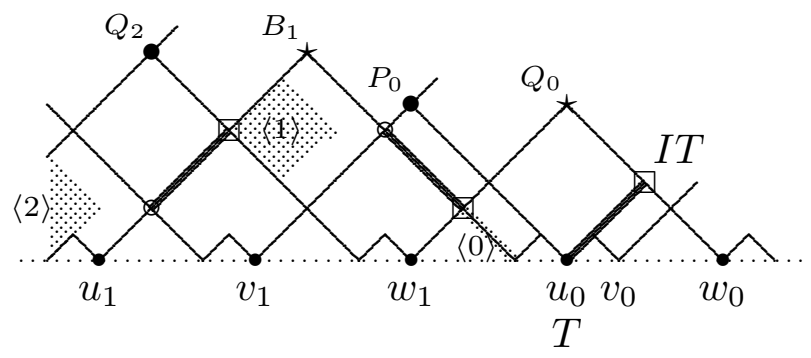


(5) If $z$ is even, then $\Sigma^{z} P S=I T$. If $z$ is odd, then $\Sigma^{z} S=I T$.

Proof. If $z$ is even, then $P S$ belongs to $\langle z\rangle$. If $z$ is odd, then $S$ belongs to $\langle z\rangle$.

(6) In order to complete the proof of (a), we still have to show: If $z$ is even, $\Omega^{z} I T=P S$ and $\operatorname{pd} T \geq z$, then id $S \geq z$. We can assume that $z=2 t \geq 2$. WAs we have seen in (2), $\Omega^{z-1} I T=\left[w_{t}, u_{t-1}\left[\right.\right.$ and $S=\tau w_{t}=\tau\left(\operatorname{soc} \Omega^{z-1}\right)$. Since $\Omega^{z-1} I T$ is not projective, the module $\left[\tau w_{t}, u_{t-1}\right.$ [ exists: it is the middle term of an element in $\operatorname{Ext}^{1}\left(\Omega^{z-1} I T, S\right)$. This shows that $\left.\operatorname{Ext}^{z}(I T, S)=\Omega^{z-1} I T, S\right) \neq 0$.

For assertion (b), it remains to show: If $z$ is odd, $\Omega^{z} T=P S$ and $\operatorname{pd} I T \geq z$, then id $P S \geq z$. But this is trivial. Namely, $\Omega^{z} T=P S$ implies that $\operatorname{Ext}^{z}(T, P S) \neq 0$, thus id $P S \geq z$.

This completes the proof of Theorem 4.1 in case $A$ is a linear Nakayama algebra.

5.2. Remark. We use the previous proof in order to mention the following observation. Recall that for a representation-finite algebra $A$, an indecomposable module $X$ is a predecessor of an indecomposable module $Y$, provided there is a path in the AuslanderReiten quiver starting in $X$ and ending in $Y$.

Let $A$ be a linear Nakayama algebra. Let $V \subseteq W$ be indecomposable modules and let $P_{\bullet}, Q_{\bullet}$ be minimal projective resolutions of $V$ and $W$, respectively. If both $\Omega^{2 t} V$ and $\Omega^{2 t} W$ are non-zero, then $\Omega^{2 t} W$ is a predecessor of all the modules $P_{i}, Q_{i}$ with $0 \leq i<2 t$. If both $\Omega^{2 t+1} V$ and $\Omega^{2 t+1} W$ are non-zero, then $\Omega^{2 t+1} V$ is a predecessor of all the modules $P_{i}, Q_{i}$ with $0 \leq i \leq 2 t$.

Proof. See (2) in section 5.2.

5.3. Representations of the quiver $\mathbb{Z}$. Before we look at cyclic Nakama algebras, let us consider $\mathbb{Z}$ as a quiver (its vertex set is the set $\mathbb{Z}$, and for any integer $z$, there is an arrow $z \rightarrow z-1$ ). Let $\rho$ be a set of relations (that means: paths of finite length $\geq 2$ ). The category $\mathcal{M}=\bmod (\mathbb{Z}, \rho)$ consists of the finite-dimensional representations of the quiver $\mathbb{Z}$ which satisfy the relations $\rho$. We assume that $\bmod (\mathbb{Z}, \rho)$ has enough projective and enough injective objects, thus we have projective resolutions and injective coresolutions in $\mathcal{M}$, as well as Auslander-Reiten sequences. Thus, as in the case of Nakayama algebras, we usually will specify $(\mathbb{Z}, \rho)$ by indicating the shape of the Auslander-Reiten quiver.

The category $\mathcal{M}$ is quite similar to the module category of a linear Nakayama algebra, in particular all indecomposable objects $M$ are serial; if $M$ has support $\{x, x+1, \ldots, y\}$, we again may write $M=[x, y+1[$. Of course, an object in $\mathcal{M}$ is simple iff it is of the form $[x, x+1$ [ for some vertex $x$, and by abuse of language, we will write just $x$ instead of $[x, x+1[$, provided no confusion is possible.

Let us stress that in contrast to the module category of a Nakayama algebra, given a vertex $x$, both representations $x$ and $I x$ may have infinite projective dimension. Here is a typical example:

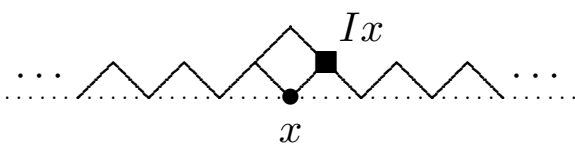

However, the analogue of Theorem 4.1 holds true in $\mathcal{M}$. 
Proposition. Let $S, T$ be simple objects in $\mathcal{M}$, and let $z \in \mathbb{N}_{0}$.

(a) If $z$ is even, $\Omega^{z} I T=P S$, and $\operatorname{pd} T \geq z$, then $\Sigma^{z} P S=I T$ and $\mathrm{id} S \geq z$.

(b) If $z$ is odd, $\Omega^{z} T=P S$, and $\operatorname{pd} I T \geq z$, then $\Sigma^{z} S=I T$ and id $P S \geq z$.

Proof. We show (a); the proof of (b) is similar. For any natural number $m$, let $A_{m}$ be the path algebra of the full subquiver $[-m, m]$ satisfying the relations in $\rho$ with support in $[-m, m]$, thus $\mathcal{M}=\bigcup_{m \in \mathbb{N}} \bmod A_{m}$.

Take minimal projective resolutions of $T$ and $I T$, say

$$
\begin{gathered}
\cdots \rightarrow P_{z} \rightarrow P_{z-1} \rightarrow \cdots \rightarrow P_{1} \rightarrow P_{0} \rightarrow T \rightarrow 0 \\
\cdots \rightarrow Q_{z} \rightarrow Q_{z-1} \rightarrow \cdots \rightarrow Q_{1} \rightarrow Q_{0} \rightarrow I T \rightarrow 0 .
\end{gathered}
$$

Since $\Omega^{z} I T=P S$, we have $Q_{z}=P S$. Choose a natural number $m \geq 1$ such that all the modules $P_{i}, Q_{i}$ with $0 \leq i \leq z$ have support in $[-m, m]$, thus they are $A_{m}$-modules, and of course projective also as $A_{m}$-modules. We have shown in 5.1 that Theorem 4.1 holds for linear Nakayama algebras, thus for $A=A_{m}$. In $\bmod A$, we have $\left(\Sigma_{A}\right)^{z} P S=I T$ and $\operatorname{id}_{A} S \geq z$. Let

$$
\begin{gathered}
0 \rightarrow S \rightarrow I_{0} \rightarrow I_{1} \rightarrow \cdots \rightarrow I_{z-1} \rightarrow\left(\Sigma_{A}\right)^{z} S \rightarrow 0 \\
0 \rightarrow P S \rightarrow J_{0} \rightarrow J_{1} \rightarrow \cdots \rightarrow J_{z-1} \rightarrow\left(\Sigma_{A}\right)^{z} P S \rightarrow 0 .
\end{gathered}
$$

be exact sequences in $\bmod A$ such that all the modules $I_{i}, J_{i}$ with $0 \leq i<z$ are indecomposable injective in $\bmod A$. It remains to be seen that the representations $I_{i}, J_{i}$ with $0 \leq i<z$ are injective in $\mathcal{M}$. According to the dual of 5.2, all these modules are predecessors of $I T$, and $I T$ is by definition injective in $\mathcal{M}$. The following quite obvious assertion finishes the proof of (a). Let I be an indecomposable injective object of $\mathcal{M}$ which is an $A_{m}$-module. Let $M$ be an indecomposable $A_{m}$-module which is a predecessor of I. If $M$ is injective as an $A_{m}$-module, then $M$ is injective in $\mathcal{M}$.

5.4. The case of $A$ being a cyclic Nakayama algebra. Let $A$ be a cyclic Nakayama algebra. In order to show (a), let $S, T$ be simple modules with $\Omega^{z} I T=P S$ and $\operatorname{pd} T \geq z$, where $z$ is even.

Let $\widetilde{A}$ be a universal cover of $A$ with push-down functor $\pi: \bmod \widetilde{A} \rightarrow \bmod A$. Note that $\bmod \widetilde{A}=\bmod (\mathbb{Z}, \rho)$ for a suitable set $\rho$ of relations. There is a natural number $b$ such that all indecomposable projective (and thus also all indecomposable injective) $A$-modules have length at most $b$. Thus mod $\widetilde{A}$ has enough projective and enough injective modules (and all indecomposable $\widetilde{A}$-modules which are projective or injective have length at most $b)$. This shows that Proposition 5.3 holds for $\bmod \widetilde{A}$.

Fix an $\widetilde{A}$-module $\widetilde{T}$ with $\pi(\widetilde{T})=T$. Let $I \widetilde{T}$ be an injective envelope of $\widetilde{T}$ (as an $\widetilde{A}$-module). Then $\pi(I \widetilde{T})=I T$. Let

$$
\begin{gathered}
\cdots \rightarrow \widetilde{P}_{z} \rightarrow \widetilde{P}_{z-1} \rightarrow \cdots \rightarrow \widetilde{P}_{1} \rightarrow \widetilde{P}_{0} \rightarrow \widetilde{T} \rightarrow 0 \\
\cdots \rightarrow \widetilde{Q}_{z} \rightarrow \widetilde{Q}_{z-1} \rightarrow \cdots \rightarrow \widetilde{Q}_{1} \rightarrow \widetilde{Q}_{0} \rightarrow I \widetilde{T} \rightarrow 0
\end{gathered}
$$


be minimal projective resolutions of $\widetilde{T}$ and $I \widetilde{T}$ (as $\widetilde{A}$-modules). Applying $\pi$ to these resolutions we obtain minimal projective resolutions of $T$ and $I T$ (as $A$-modules), respectively. Since pd $I T=z$, we see that the map $\widetilde{Q}_{z} \rightarrow \widetilde{Q}_{z-1}$ is injective and that $\pi\left(\widetilde{Q}_{z}\right)=\Omega^{z} I T=P S$. In particular, $\widetilde{Q}_{z}$ is indecomposable projective, say equal to $P \widetilde{S}$ with $\widetilde{S}$ a simple $\widetilde{A}$-module, and we have $\pi(\widetilde{S})=S$.

Altogether, we see: $\widetilde{S}$ and $\widetilde{T}$ are simple $\widetilde{A}$-modules with $\Omega^{z} I \widetilde{T}=P \widetilde{S}$ and $\operatorname{pd} \widetilde{T} \geq z$ (and $z$ is even). Proposition 5.3 (a) asserts that $\Sigma^{z} P \widetilde{S}=I \widetilde{T}$ and that id $\widetilde{S} \geq z$. Now using the push-down functor $\pi$, it follows that $\Sigma^{z} P S=I T$ and that id $S \geq z$.

This completes the proof of (a). The proof of (b) is, of course, similar.

This completes the proof of Theorem 4.1.

5.5. An example. Let $A$ be a linear Nakayama algebra, and $S, T$ are simple modules, with $P S=\Omega^{z} I T$ and $\Sigma^{z} P S$, where $z=2 t$ is even. Let $P_{\bullet}$ be a minimal projective resolution of $I T$, and $I \bullet$ a minimal injective coresolution of $P S$, then these two sequences yield non-zero elements of $\operatorname{Ext}^{z}(I T, P S)$.

We present one example in detail, here is $z=4$, thus $t=2$. There is a commutative diagram of the following form

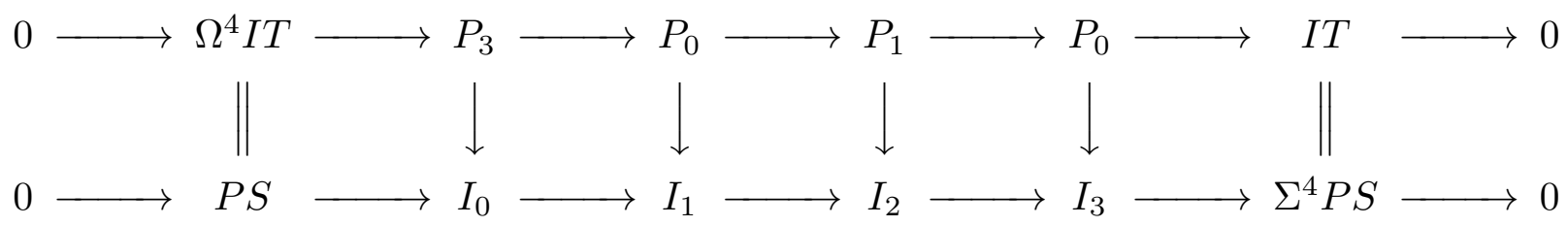

which yields canonical maps $\Omega^{i} I T \rightarrow \Sigma^{z-i} P S$.

For the example which we have selected, the map $\Omega^{2} I T \rightarrow \Sigma^{2} P S$ is neither a monomorphism nor an epimorphism:

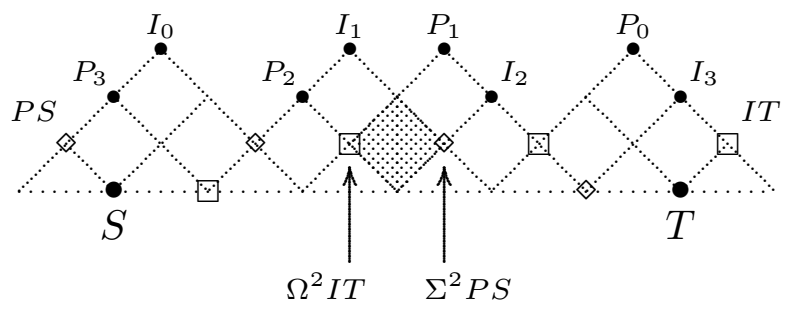

Appendix A. The functions $\psi$ and $\gamma$.

We assume that $A$ is a cyclic Nakayama algebra.

A.1. Proposition. Let $A$ be a cyclic Nakayama algebra. For all $t \geq 0$, we have

$$
\psi^{t} \gamma^{t} \psi^{t}=\psi^{t} \quad \text { and } \quad \gamma^{t} \psi^{t} \gamma^{t}=\gamma^{t} .
$$

The proof will be given in A.4, using some basic properties of monotone endofunctions of $\mathbb{Z}$, see A.3. 
Corollary 1. Let $A$ be a cyclic Nakayama algebra. For all $t \geq 0$, the map

$$
\gamma^{t}: \operatorname{Im} \psi^{t} \rightarrow \operatorname{Im} \gamma^{t}
$$

is a bijection with inverse $\psi^{t}$. In particular, we have $\left|\operatorname{Im} \psi^{t}\right|=\left|\operatorname{Im} \gamma^{t}\right|$.

Recall that $a(A)$ is the smallest natural number $a$ with $\operatorname{Im} \psi^{a}=\operatorname{Im} \psi^{a+1}$. Using duality, the smallest natural number $b$ with $\operatorname{Im} \gamma^{b}=\operatorname{Im} \gamma^{b+1}$ is $a\left(A^{\text {op }}\right)$.

We denote by $c(A)$ the number of $\psi$-cyclic modules. Thus, using duality, $c\left(A^{\mathrm{op}}\right)$ is the number of $\gamma$-cyclic $A$-modules.

Corollary 2. Let $A$ be a cyclic Nakayama algebra. Then

$$
a(A)=a\left(A^{\mathrm{op}}\right) \quad \text { and } \quad c(A)=c\left(A^{\mathrm{op}}\right) .
$$

Proof. We have $\operatorname{Im} \psi^{0} \supseteq \operatorname{Im} \psi^{1} \supseteq \operatorname{Im} \psi^{2} \supseteq \cdots$. If $S$ is a simple module, then, by definition, $a(S)=t$ provided $S$ belongs to $\operatorname{Im} \psi^{t-1} \backslash \operatorname{Im} \psi^{t}$, and $a(S)=\infty$ provided $S$ belongs to $\bigcap_{t} \operatorname{Im} \psi^{t}$.

Similarly, $\operatorname{Im} \gamma^{0} \supseteq \operatorname{Im} \gamma^{1} \supseteq \operatorname{Im} \gamma^{2} \supseteq \cdots$. If $S$ is a simple module, let $a^{\prime}(S)=t$ provided $S$ belongs to $\operatorname{Im} \gamma^{t-1} \backslash \operatorname{Im} \gamma^{t}$, and let $a^{\prime}(S)=\infty$ provided $S$ belongs to $\bigcap_{t} \operatorname{Im} \gamma^{t}$. The simple module $S$ is $\gamma$-cyclic, provided $S$ belongs to $\operatorname{Im} \gamma^{t}$ for all $t$, thus provided $a^{\prime}(S)=\infty$. Using duality, we see that $a\left(A^{\mathrm{op}}\right)$ is the maximum of the numbers $a^{\prime}(S)$, where $S$ is simple and not $\gamma$-cyclic.

Since $\gamma^{t}$ provides a bijection between $\operatorname{Im} \psi^{t}$ and $\operatorname{Im} \gamma^{t}$, it provides a bijection from the set of isomorphism classes $[S]$ of simple modules with $a(S) \geq t$ onto the set of isomorphism classes $[S]$ of simple modules with $a^{\prime}(S) \geq t$. It follows that $a(A)=a\left(A^{\mathrm{op}}\right)$.

For $t \geq a(A)$, the map $\gamma^{t}$ provides a bijection from the set of $\psi$-cyclic simple modules onto the set of $\gamma$-cyclic modules. This shows that $c(A)=c\left(A^{\mathrm{op}}\right)$.

A.2. Proposition. Let $A$ be a cyclic Nakayama algebra. For all $t \geq 0$, we have

$$
\psi^{t} \tau^{-} \gamma^{t} \tau \psi^{t}=\psi^{t} \quad \text { and } \quad \gamma^{t} \tau \psi^{t} \tau^{-} \gamma^{t}=\gamma^{t} .
$$

The proof will be similar to the proof of Proposition A.1, see A.5. As in A.1, there is the following Corollary.

Corollary. Let $A$ be a cyclic Nakayama algebra. For all $t \geq 0$, the map

$$
\gamma^{t} \tau: \operatorname{Im} \psi^{t} \rightarrow \operatorname{Im} \gamma^{t}
$$

is a bijection with inverse $\psi^{t} \tau^{-}$.

Proof. The image of $\gamma^{t} \tau$ is contained in $\operatorname{Im} \gamma^{t}$, thus $\gamma^{t} \tau$ maps $\operatorname{Im} \psi^{t}$ into $\operatorname{Im} \gamma^{t}$. Similarly, $\psi^{t} \tau^{-}$maps $\operatorname{Im} \gamma^{t}$ into $\operatorname{Im} \psi^{t}$. The equalities in A.2 yield the assertion.

A.3. A (set-theoretical) function $f: \mathbb{Z} \rightarrow \mathbb{Z}$ is said to be monotone provided $i<j$ in $\mathbb{Z}$ implies $f(i) \leq f(j)$. 
Lemma. Let $f, g: \mathbb{Z} \rightarrow \mathbb{Z}$ be monotone functions with $f g(i) \geq i \geq g f(i)$ for all $i \in \mathbb{Z}$. Then, for all $t \geq 0$, we have $f^{t} g^{t}(i) \geq i \geq g^{t} f^{t}(i)$, and $f^{t} g^{t} f^{t}=f^{t}$ and $g^{t} f^{t} g^{t}=g^{t}$.

Proof. First, we show by induction on $t$ that $f^{t} g^{t}(i) \geq i$ for all $i \in \mathbb{Z}$. The case $t=1$ is one of the assumptions. Thus, assume that we know already for some $t$ that $f^{t} g^{t}(i) \geq i$ for all $i$. Replacing $i$ by $g(i)$, we obtain $f^{t} g^{t+1}(i) \geq g(i)$. Since $f$ is monotone, we get $f^{t+1} g^{t+1}(i) \geq f g(i)$. Altogether we see that $f^{t+1} g^{t+1}(i) \geq f g(i) \geq i$. Similarly, we see that $i \geq g^{t} f^{t}(i)$ for all $i$, and all $t \geq 0$.

Applying $f^{t}$ to $i \geq g^{t} f^{t}(i)$, the monotony gives $f^{t}(i) \geq f^{t} g^{t} f^{t}(i)$. On the other hand, we take $f^{t} g^{t}(i) \geq i$ and replace $i$ by $f^{t}(i)$, this yields $f^{t} g^{t} f^{t}(i) \geq f^{t}(i)$. Altogether we have $f^{t} g^{t} f^{t}(i) \geq f^{t}(i) \geq f^{t} g^{t} f^{t}(i)$, thus $f^{t} g^{t} f^{t}=f^{t}$. Similarly, we see that $g^{t} f^{t} g^{t}=g^{t}$.

A.4. Now, let $A$ be a cyclic Nakayama algebra. In order to use A.3, we need to work with the universal cover $\widetilde{A}$ of the algebra $A$ and a covering functor $\pi: \bmod \widetilde{A} \rightarrow \bmod A$ (see $[\mathrm{Ga}]$ and related papers by Bongartz-Gabriel and Gordon-Green). The quiver of $\widetilde{A}$ is $\mathbb{Z}$ (we consider the integers $\mathbb{Z}$ as a quiver with vertex set $\mathbb{Z}$ and with arrows $i \rightarrow i-1$ for all $i \in \mathbb{Z}$ ). We have to fix a simple module $S=S(0)$, define $S(i)=\tau^{-i} S$ and use as covering map $\pi: i \mapsto S(i)$. Note that the Auslander-Reiten quiver of $\widetilde{A}$ is a full subquiver of the translation quiver $\mathbb{Z A}_{\infty}$, its lower boundary is formed by the simple modules. We should stress that the integers which index the simple modules increase when going from right to left.

Let $\widetilde{\psi}(i)=i+|I(i)|$ and $\widetilde{\gamma}(i)=i-|P(i)|$. Then $\widetilde{\psi}$ is a covering of $\psi$, and $\widetilde{\gamma}$ is a covering of $\gamma$ (this means: $\pi \widetilde{\psi}=\psi \pi$ and $\pi \widetilde{\gamma}=\gamma \pi$ ). Similarly, let $\widetilde{\tau}(i)=i-1$; thus $\widetilde{\tau}$ is a covering of $\tau$.

(1) The functions $\widetilde{\psi}$ and $\widetilde{\gamma}$ are monotone.

Proof. Let $i<j$ in $\mathbb{Z}$. If $j-i \geq|I(i)|$, then $\widetilde{\psi}(i)=i+|I(i)| \leq j<j+|I(j)|=\widetilde{\psi}(j)$. Thus, we can assume that $j-i<|I(i)|$. Since $0<j-i<|I(i)|$, there is a submodule $U$ of $I(i)$ of length $j-i$ and $I(i) / U$ has socle $j$.

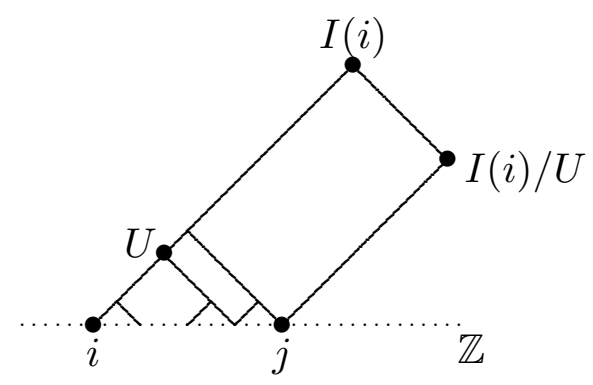

Since $I(i) / U$ is a module with socle $j$, it is a submodule of $I(j)$, thus $|I(j)| \geq|I(i) / U|=$ $|I(i)|-|U|=|I(i)|-j+i$. This shows that $\widetilde{\psi}(i)=i+|I(i)| \leq j+|I(j)|=\psi(j)$. This shows that $\widetilde{\psi}$ is monotone.

In the same way, or using duality, one sees that $\widetilde{\gamma}$ is monotone.

(2) If $i \in \mathbb{Z}$, then $\widetilde{\psi} \widetilde{\gamma} i \leq i \leq \widetilde{\gamma} \widetilde{\psi}$. 
Proof. We show that $|I(i)| \geq|P(\widetilde{\psi} i)|$. Assume, for the contrary, that $|I(i)|<|P(\widetilde{\psi} i)|$. Then $P(\widetilde{\psi} i)$ has a submodule $V$ of length $|I(i)|+1$. Note that the socle of $V$ has to be $i$.

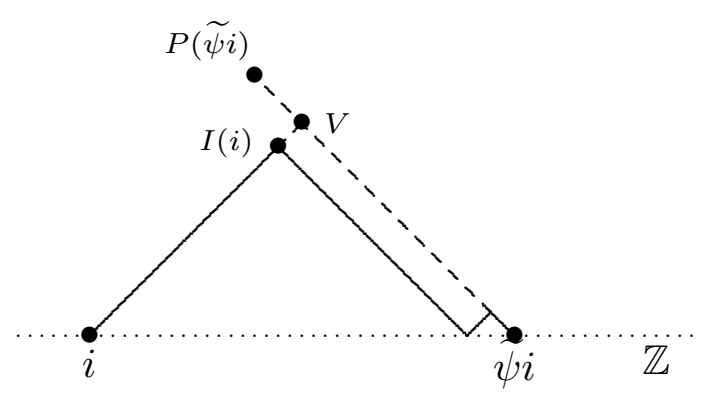

This implies that $I(i)$ is a submodule of $V$, and, of course, a proper submodule. This is impossible, since $I(i)$ is injective.

Since $|I(i)| \geq|P(\widetilde{\psi} i)|$, we see that $i \leq i+|I(i)|-|P(\widetilde{\psi} i)|=\widetilde{\psi}(i)-|P(\widetilde{\psi} i)|=\widetilde{\gamma} \widetilde{\psi}(i)$. In the same way, or using duality, one sees that $\widetilde{\psi} \widetilde{\gamma} i \leq i$.

Proof of Proposition A.1. The assertions (1) and (2) show that we can apply Lemma A.3 to the functions $f=\widetilde{\psi}$ and $g=\widetilde{\gamma}$. We get $\widetilde{\psi}^{t} \widetilde{\gamma}^{t} \widetilde{\psi}^{t}=\widetilde{\psi}^{t}$ as well as $\widetilde{\gamma}^{t} \widetilde{\psi}^{t} \widetilde{\gamma}^{t}=\widetilde{\gamma}^{t}$, for all $t \geq 0$. But $\widetilde{\psi}^{t} \widetilde{\gamma}^{t} \widetilde{\psi}^{t}=\widetilde{\psi}^{t}$ implies that $\psi^{t} \gamma^{t} \psi^{t}=\psi^{t}$. Similarly, $\widetilde{\gamma}^{t} \widetilde{\psi}^{t} \widetilde{\gamma}^{t}=\widetilde{\gamma}^{t}$ implies that $\gamma^{t} \psi^{t} \gamma^{t}=\gamma^{t}$.

A.5. As a second application of A.3, we show Proposition A.2.

(1) The function $\widetilde{\tau}^{-} \widetilde{\gamma} \widetilde{\tau}$ is monotone.

Proof. This follows directly from A.2, Lemma 1 and the fact that $\widetilde{\tau}$ and $\widetilde{\tau}^{-}$are, of course, monotone.

(2) If $i \in \mathbb{Z}$, then $\widetilde{\tau}^{-} \widetilde{\gamma} \widetilde{\psi} \widetilde{\psi}(i) \leq i \leq \widetilde{\psi} \widetilde{\tau}^{-} \widetilde{\gamma} \widetilde{\tau}(i)$.

Proof. First, let us show that $\widetilde{\tau}^{-} \widetilde{\gamma} \widetilde{\psi} \widetilde{\psi}(i) \leq i$. Let $j=\widetilde{\tau} \widetilde{\psi}(i)$. Always, $I(i)$ is a factor module of $P(j)$ and $x=\widetilde{\tau}^{-} \gamma(j)$ is the socle of $P(j)$. Thus $x \leq i$.

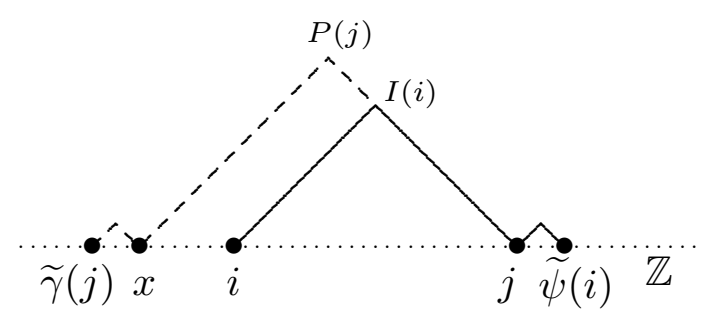

Second, we want to show that $i \leq \widetilde{\psi} \widetilde{\tau}^{-} \widetilde{\gamma} \widetilde{\tau}(i)$. We start with $i$ and $\widetilde{\tau}(i)=i-1$ and let $j=\widetilde{\tau}^{-} \widetilde{\gamma}(i-1)$. Note that $j=\operatorname{soc} P(i-1)$. It follows that $P(i-1) \subseteq I(j)$. If $P(i-1)=I(j)$, then $i=\widetilde{\psi}(j)$. Otherwise, $P(i-1)$ is a proper submodule of $I(j)$ and then $i<\widetilde{\psi}(j)$.

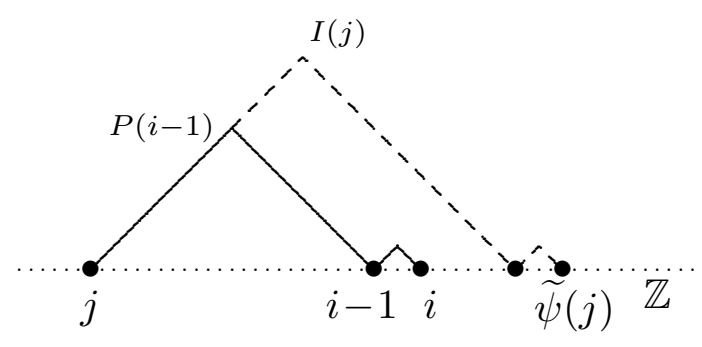


Proof of Proposition A.2. The functions $f=\widetilde{\tau}^{-} \widetilde{\gamma} \widetilde{\tau}$ and $g=\widetilde{\psi}$ are monotone, according to A.5 (1) and A.4 (1), respectively. According to A.5 (2), we see that the functions $f$ and $g$ satisfy the conditions of Lemma A.3. Thus, for all $t \geq 0$, we have $\psi^{t} \tau^{-} \gamma^{t} \tau \psi^{t}=$ $\psi^{t}\left(\tau^{-} \gamma \tau\right)^{t} \psi^{t}=\psi^{t}$ and $\tau^{-} \gamma^{t} \tau \psi^{t} \tau^{-} \gamma^{t} \tau=\left(\tau^{-} \gamma \tau\right)^{t} \psi^{t}\left(\tau^{-} \gamma \tau\right)^{t}=\left(\tau^{-} \gamma \tau\right)^{t}=\tau^{-} \gamma^{t} \tau$. Multiplying the latter equality from the left by $\tau$, from the right by $\tau^{-}$, we get $\gamma^{t} \tau \psi^{t} \tau^{-} \gamma^{t}=\gamma^{t}$, as required.

A.6. The case $t=1$. Note that $\operatorname{Im} \psi$ are just the simple modules with projective dimension greater than 1 . Similarly, $\operatorname{Im} \gamma$ are just the simple modules with injective dimension greater than 1 .

There is the bijection

$$
\gamma: \operatorname{Im} \psi=\{T \mid \operatorname{pd} T \geq 2\} \longrightarrow \operatorname{Im} \gamma=\{S \mid \text { id } S \geq 2\}
$$

with inverse $\psi$ :

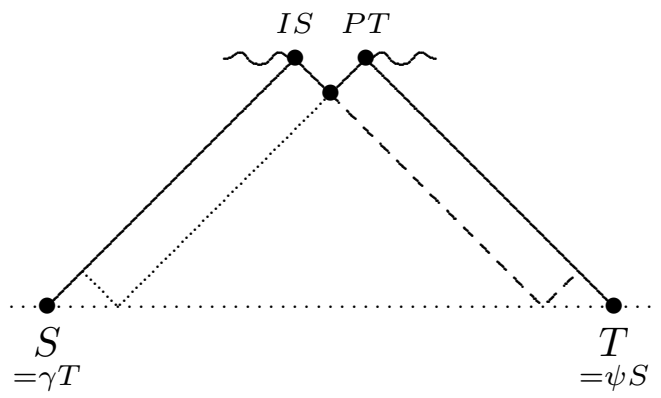

This bijection between $\operatorname{Im} \psi=\{T \mid \operatorname{pd} T \neq 1\}$ and $\operatorname{Im} \gamma=\{S \mid$ id $S \neq 1\}$ can also be seen also as bijections of $\operatorname{Im} \psi$ and $\operatorname{Im} \gamma$ with the set of "valleys" of the "roof" of $A$ (see [R2]); by definition, a module $V$ is called a valley provided $V$ is both the radical of a projective module as well of the form $I S / S$, where $S$ is simple. Corresponding to the valleys in the roof, there are the peaks (the indecomposable modules which are both projective and injective).

There is also the bijection

$$
\gamma \tau: \operatorname{Im} \psi=\{T \mid \operatorname{pd} T \geq 2\} \longrightarrow \operatorname{Im} \gamma=\{S \mid \operatorname{id} S \geq 2\}
$$

with inverse $\psi \tau^{-}$

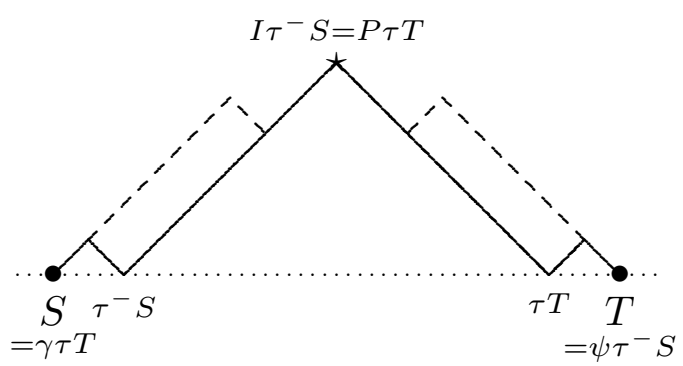

Here, the middle module $I \tau^{-} S=P \tau T$ is a peak and $\gamma \tau$ provides bijections between $\{T \mid \operatorname{pd} T \geq 2\}$ and the set of peaks as well as between the set of peaks and $\{S \mid$ id $S \geq 2\}$. 
Note that all these sets $\{T \mid \operatorname{pd} T \geq 2\},\{S \mid$ id $S \geq 2\}$, the set of valleys and the set of peaks have the same cardinality as a minimal set of admissible relations which are needed to define $A$, see Appendix C.

A.7. Historical remark. Some of the effects of the interrelation between $\psi$ and $\gamma$ were already discussed by Shen [Sh3]. The assertion that there are as many $\psi$-cyclic simple modules as there are $\gamma$-cyclic simple modules was shown by Shen in [Sh2].

\section{A.8. The $\psi$-quiver and the $\gamma$-quiver of a cyclic Nakayama algebra $A$.}

Using $\gamma$, one can define the resolution quiver (or $\gamma$-quiver) of $A$, see [R2]: its vertices are the isomorphism classes $[S]$ of the simple modules $S$, and there is an arrow $[S] \rightarrow[T]$ provided $T=\gamma(S)$. Of course, the $\gamma$-paths are just the paths in the resolution quiver.

Dually, using $\psi$, one can define the coresolution quiver (or $\psi$-quiver) of $A$ : its vertices are the isomorphism classes $[S]$ of the simple modules $S$, and there is an arrow $[S] \rightarrow[T]$ provided $T=\psi(S)$. The $\psi$-paths are just the paths in the coresolution quiver.

Note that the coresolution quiver of $A$ is just the resolution quiver of $A^{\mathrm{op}}$.

Warning. The $\psi$-quiver and the $\gamma$-quiver of a Nakayama algebra $A$ have many properties in common. Several such properties are mentioned above. In addition, Shen [Sh1] has shown, that also the number of components are equal. However, the cardinalities of the components of the $\gamma$-quiver may be different from the cardinalities of the components of the $\psi$-quiver as the following example shows:
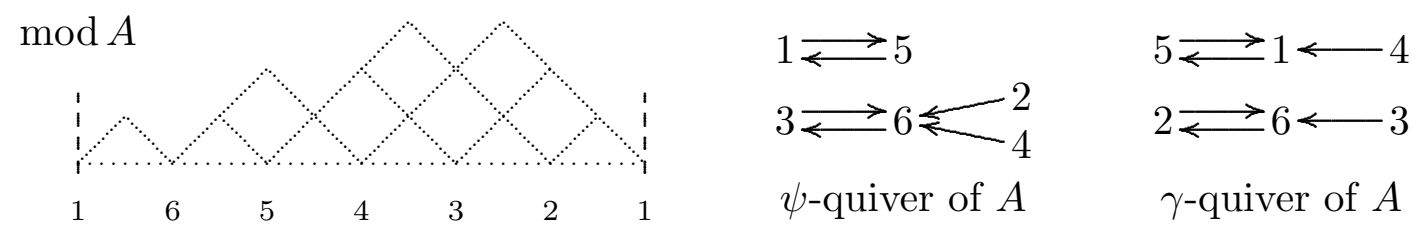

Remark. The $\psi$-quiver (and similarly, the $\gamma$-quiver) of a cyclic Nakayama algebra $A$ do not determine the finitistic dimension of $A$. Namely, the following two algebras $A, A^{\prime}$ have the same $\psi$-quiver, but fin-pro $A=2$, whereas fin-pro $A^{\prime}=1$.
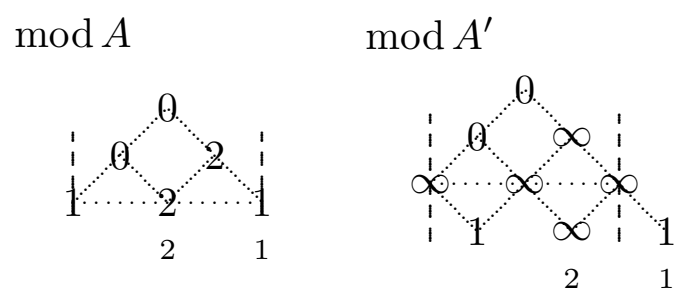

$\psi$-quiver

\section{Appendix B. Sen's $\epsilon$-construction.}

It seems to be worthwhile to add a short outline of a reduction method which was introduced and very skillfully used by Sen [S1, S2, S3], since it relates to the functions $\gamma$ and $\psi$ (discussed in sections 3 and 4 , as well as in Appendix A).

Let $A$ be a cyclic Nakayama algebra. Sen considers the subcategory $\mathcal{F}$ of modules $M$ with what he calls "syzygy filtrations": this is an abbreviation of saying that $M$ has a filtration whose factors are second syzygies of simple modules (denoted below by $\Delta T$ ). 
B.1. Three sets of simple modules will play a role: $\mathcal{S}, \mathcal{T}$ and $\mathcal{U}$ (see also Appendix C). Here, $\mathcal{S}$ is the set of simple torsionless modules. Note that an indecomposable module $M$ is torsionless if and only if soc $M$ belongs to $\mathcal{S}$. Then, $\mathcal{T}$ is the set of simple modules $T$ with id $T \geq 2$; these are the simple modules in $\operatorname{Im} \gamma$. Finally, $\mathcal{U}$ is the set of simple modules $U$ with id $U \geq 2$; these are the simple modules in $\operatorname{Im} \psi$. A simple module $S$ belongs to $\mathcal{S}$ if and only if $\tau S$ belongs to $\mathcal{T}$.

Let $\mathcal{F}$ be the full subcategory of all modules with top in add $\mathcal{T}$ and socle in add $\mathcal{S}$. Thus, an indecomposable module $M$ belongs to $\mathcal{F}$ if and only if top $M$ belongs to $\mathcal{T}$ and $M$ is torsionless.

B.2. The modules $\Delta T$. For $T \in \mathcal{T}$, let $\Delta T$ be the smallest non-zero factor module of $P T$ which is torsionless (since $P T$ is torsionless, $\Delta T$ exists; also, $\Delta T$ is, of course, uniquely determined by $T$ ).

(a) The module $\Delta T$ has a unique composition factor which belongs to $\mathcal{T}$, namely $T=$ top $\Delta T$. Similarly, $\Delta T$ has a unique composition factor which belongs to $\mathcal{S}$, namely $\tau^{-} T=$ $\operatorname{soc} \Delta T$.

(b) If $C$ is any simple module, there is a unique $T \in \mathcal{T}$ such that $C$ is a composition factor of $\Delta T$ and the multiplicity of $C$ in $\Delta T$ is 1 . (Thus, every simple module occurs with multiplicity 1 as a composition factor of $\bigoplus_{T \in \mathcal{T}} \Delta T$.)

(c) If $T \in \mathcal{T}$, then

$$
\Delta T=\Omega^{2} \psi T,
$$

thus $\Delta T=\Omega^{2} U$, where $U=\psi T \in \mathcal{U}$.

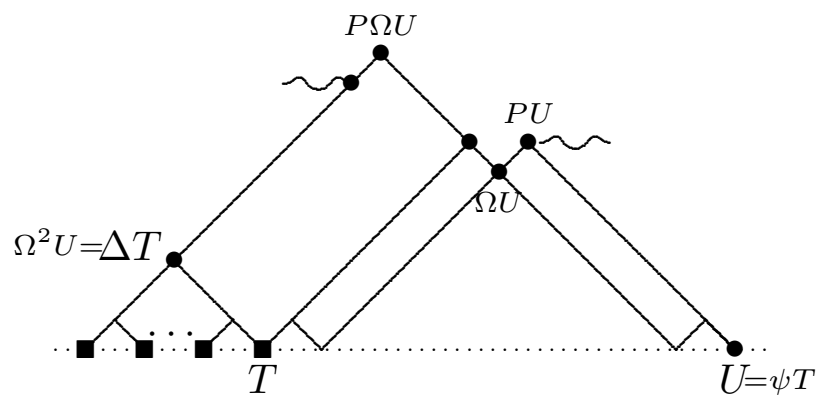

Conversely, if $U$ is simple, then either $\operatorname{pd} U \leq 1$ and $\Omega^{2} U=0$, or else $U \in \mathcal{U}$, and then $T=\gamma U$ belongs to $\mathcal{T}$ and $\psi T=U$. It follows that the modules $\Delta T$ are just the non-zero modules which are second syzygy modules of simple modules.

Note that the relevance of the modules $\Omega^{2} U$ mentioned in (c) was stressed already by Madsen $[\mathrm{M}]$.

Proof. (a) First, we show that top $\Delta T$ is the only composition factor of $\Delta T$ which belongs to $\mathcal{T}$. Namely, assume, for the contrary, that there are submodules $X^{\prime} \subset X \subset \Delta T$ with $X / X^{\prime} \in \mathcal{T}$. Then $\operatorname{soc}(\Delta T / X) \simeq \tau^{-} \operatorname{top} X=\tau^{-}\left(X / X^{\prime}\right)$ is in $\mathcal{S}$. This implies that $\Delta T / X$ is torsionless. But this contradicts the minimality of $\Delta T$.

Next, we show that soc $\Delta T$ is the only composition factor of $\Delta T$ which belongs to $\mathcal{S}$. If $0 \subset X^{\prime} \subset X \subseteq \Delta T$ are submodules such that $X / X^{\prime}$ is in $\mathcal{S}$, then top $X^{\prime} \simeq \tau \operatorname{soc}\left(\Delta T / X^{\prime}\right)=$ $\tau\left(X / X^{\prime}\right)$ belongs to $\mathcal{T}$. But this contradicts the first assertion. 
(b) Let $S$ be simple. Let $c \geq 1$ be minimal with $\tau^{-c} S$ torsionless. Thus $T=\tau^{-c+1} S=$ $\tau\left(\tau^{-c} S\right)$ belongs to $\mathcal{T}$, whereas the modules $\tau^{i} T$ with $1 \leq i<c$ do not belong to $\mathcal{T}$. It follows that $\Delta T$ has length at least $c$ and that $S$ is a composition factor of $\Delta T$. Since the top $T$ of $\Delta T$ has Jordan-Hölder multiplicity 1, any composition factor of $\Delta T$ has multiplicity 1 .

(c) Let $T \in \mathcal{T}$ and $U=\psi T$. Then $\operatorname{pd} U \geq 2$ and $\gamma U=T$. Since $\operatorname{pd} U \geq 2$, it follows from 3.2 (b) that top $\Omega^{2} U=\gamma U=T$. Of course, $\Omega^{2} U$ is torsionless, thus we see that $\Delta T$ is a factor module of $\Omega^{2} U$. According to 3.2 (c), only the socle of $\Omega^{2} U$ can be torsionless, thus $\Delta T=\Omega^{2} U$.

Conversely, let $U$ be simple. If $\operatorname{pd} U \leq 1$, then $\Omega^{2} U=0$. Thus, let $\operatorname{pd} U \geq 2$ and $T=\gamma U$. Thus, $T \in \mathcal{T}$ and $T=\gamma U$.

B.3. The category $\mathcal{F}$. The modules $\Delta T$ with $T \in \mathcal{T}$ are pairwise orthogonal bricks and $\mathcal{F}$ is the full subcategory of all modules which have a filtration with factors of the form $\Delta T$ with $T \in \mathcal{T}$.

The category $\mathcal{F}$ is an extension-closed exact abelian category with simple objects the modules $\Delta T$ with $T \in \mathcal{T}$. The category $\mathcal{F}$ is a serial length category with

$$
\tau_{\mathcal{F}} \Delta T=\Delta(\tau \operatorname{soc} \Delta T),
$$

provided $\Delta T$ is not projective. (Here, $\tau_{\mathcal{F}}$ is the Auslander-Reiten translation inside the category $\mathcal{F}$.)

Proof. Let $T \in \mathcal{T}$. If $f: \Delta T \rightarrow \Delta T$ is a non-zero non-invertible endomorphism, then the image of $f$ is a proper submodule of $\Delta T$ with top $T$, a contradiction. Also, if $T^{\prime}, T$ are non-isomorphic modules in $\mathcal{T}$, and $f: \Delta T^{\prime} \rightarrow \Delta T$ is a non-zero homomorphism, then the image of $f$ is a submodule of $\Delta T$ with top $T^{\prime}$, again a contradiction. Thus, we see that the modules $\Delta T$ with $T \in \mathcal{T}$ are pairwise orthogonal bricks.

Next, we show that an indecomposable module $M$ with top in $\mathcal{T}$ and socle in $\mathcal{S}$ has a filtration with factors of the form $\Delta T$ with $T \in \mathcal{T}$. The proof is by induction on the length of $M$. Let $M$ be a module with top in $\mathcal{T}$ and socle in $\mathcal{S}$. Say let top $M=T^{\prime}$. Then $M$ is a factor module of $P T^{\prime}$. Since soc $M$ is torsionless, also $M$ is torsionless. By definition of $\Delta T^{\prime}$ we see that $\Delta T^{\prime}$ is a factor module of $P T^{\prime}$, say $M=P T^{\prime} / X$ for some submodule $X$ of $P T^{\prime}$. Now top $X=\tau \operatorname{soc}\left(P T^{\prime} / X\right)=\tau \operatorname{soc} M$, thus top $X$ is in $\mathcal{T}$. By induction, $X$ has a filtration with factors of the form $\Delta T$.

It follows that $\mathcal{F}$ is the extension closure of the class of modules $\Delta T$ with $T \in \mathcal{T}$, and that $\mathcal{F}$ is an extension-closed exact abelian subcategory, whose simple objects are just the objects $\Delta T$ with $T \in \mathcal{T}$, see for example [R1].

It remains to calculate $\tau_{\mathcal{F}} \Delta T$. Let $\pi: P T \rightarrow \Delta T$ be a projective cover. If $\Delta T$ is not projective, then $\operatorname{Ker}(f)$ is an indecomposable module, which belongs to $\mathcal{F}$ and has $\Delta T^{\prime}$ with $T^{\prime}=\tau \operatorname{soc} \Delta T$ as a factor object (its top in $\mathcal{F}$ ). Thus $\tau_{\mathcal{F}} \Delta T=\Delta\left(T^{\prime}\right)$.

Note: The algebra $\epsilon(A)$ is not necessarily cyclic, nor even connected. For example, for

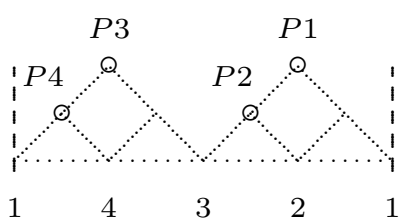


the set $\mathcal{F}$ consists of the projective modules $P 2$ and $P 4$. Thus, $\mathcal{F}$ is a semisimple category and its quiver consists of two singletons (and $\epsilon(A)=k \times k)$.

The subcategory $\mathcal{F}$ of $\bmod A$ is closed under projective covers. It follows that the abelian category $\mathcal{F}$ has enough projective objects. The indecomposable projective objects in $\mathcal{F}$ are the modules $P T$ with $T \in \mathcal{T}$.

Proof that $\mathcal{F}$ is closed under projective covers: if $M$ is indecomposable and in $\mathcal{F}$, say with top $T$, then $P M=P T$ also belongs to $\mathcal{F}$.

The module $G=\bigoplus_{T \in \mathcal{T}} P T$ is a progenerator of $\mathcal{F}$. It follows that $\epsilon(A)=(\text { End } G)^{\text {op }}$ is a Nakayama algebra and there is a categorical equivalence

$$
\mathcal{F} \simeq \bmod \epsilon(A)
$$

B.4. The $\gamma$-quiver of $\epsilon(A)$. We assume that none of the modules $\Delta T$ is projective, thus $\epsilon(A)$ is again a cyclic Nakayama algebra. Using the categorical equivalence $\mathcal{F} \simeq$ $\bmod \epsilon(A)$, the $\gamma$-quiver of $\epsilon(A)$ may be considered as a quiver with vertex set the set of modules $\Delta T$ with $T \in \mathcal{T}$ and with arrows $\Delta T \rightarrow \gamma_{\mathcal{F}}(\Delta T)$. There is the following observation:

Lemma. If $T \in \mathcal{T}$, then

$$
\gamma_{\mathcal{F}}(\Delta T)=\Delta(\gamma T)
$$

Proof. Let $\operatorname{soc}_{\mathcal{F}} P_{\mathcal{F}}(\Delta T)=\Delta\left(T^{\prime}\right)$ for some $T^{\prime}$. Then $\operatorname{soc} \Delta\left(T^{\prime}\right)=\operatorname{soc} P T$. Since $\gamma_{\mathcal{F}}=\tau_{\mathcal{F}} \operatorname{soc}_{\mathcal{F}} P_{\mathcal{F}}(-)$ and $\gamma=\tau \operatorname{soc} P(-)$, we see that

$$
\begin{aligned}
\gamma_{\mathcal{F}}(\Delta T) & =\tau_{\mathcal{F}} \operatorname{soc}_{\mathcal{F}} P_{\mathcal{F}}(\Delta T) \\
& =\tau_{\mathcal{F}} \Delta\left(T^{\prime}\right)=\Delta\left(\tau \operatorname{soc} \Delta\left(T^{\prime}\right)\right) \\
& =\Delta(\tau \operatorname{soc} P T)=\Delta(\gamma T) .
\end{aligned}
$$

This shows: Let $\Gamma(A)$ be the $\gamma$-quiver of $A$ and $\Gamma(A) \mid \operatorname{Im} \gamma$ its restriction to $\operatorname{Im} \gamma$. Then the map $T \mapsto \Delta(T)$ identifies $\Gamma(A) \mid \operatorname{Im} \gamma$ with the $\gamma_{\mathcal{F}}$-quiver of $\epsilon(A)$.

\section{B.5. The subcategory $\mathcal{F}$ and the class of reflexive modules.}

Proposition. The modules in $\mathcal{F}$ are reflexive.

Proof. Assume that $M$ belongs to $\mathcal{F}$. We want to show that $M$ is reflexive. We can assume that $M$ is indecomposable and not projective. Now $M$ is torsionless, thus $I M$ is projective. Let $0 \neq P \subseteq I M$ be minimal projective (this means: projective, and $\operatorname{rad} P$ is not projective). Since $M$ is a non-projective submodule of $I M$, we see that $M$ is a proper submodule of $P$. It follows that the inclusion map $u: M \rightarrow P$ is a minimal left add ${ }_{A} A$-approximation, therefore $\mho S M=\operatorname{Cok} u$. Now top $M$ belongs to $\mathcal{T}$, therefore soc $\operatorname{Cok} u=\tau^{-}$top $M$ belongs to $\mathcal{S}$, thus Cok $u$ is torsionless. It is well-known that a module $M$ is reflexive if and only if both $M$ and $\mho S M$ are torsionless (see for example [AZ], Theorem 1.5). 
Let $\mathcal{R}$ be the full subcategory of all reflexive modules. Let $\mathcal{R}_{0}$ be the full subcategory of all reduced reflexive modules (a module $M$ is said to be reduced provided $M$ has no non-zero projective direct summand).

Corollary. We have

$$
\mathcal{R}_{0} \subseteq\left\{\Omega^{2} X \mid X \in \bmod A\right\} \subseteq \mathcal{F} \subseteq\left\{P \oplus \Omega^{2} X \mid X \in \bmod A, P \in \operatorname{add}_{A} A\right\}=\mathcal{R}
$$

Proof. First, let $M$ be indecomposable, reflexive and non-projective. If $X=\mho^{2} M$, then $M=\Omega^{2} X$. This shows that $\mathcal{R}_{0} \subseteq\left\{\Omega^{2} X \mid X \in \bmod A\right\}$.

Next, let us show that any module of the form $\Omega^{2} M$ belongs to $\mathcal{F}$. On the one hand, $\Omega^{2} M$ is torsionless. On the other hand, either $\Omega^{2} M=0$, or else $\operatorname{top} \Omega^{2} M=\gamma$ top $M$, thus top $\Omega^{2} M \in \mathcal{T}$. This shows that $M \in \mathcal{F}$.

Second, let $M \in \mathcal{F}$. We want to show that $M=P \oplus \Omega^{2} X$ for some modules $P, X$ with $P$ projective. We can assume that $M$ is indecomposable and not projective. As we saw, $M$ is reflexive. Thus, $M=\Omega^{2} X$ for some module $X$.

Examples. All three inclusions mentioned in the Corollary may be proper.
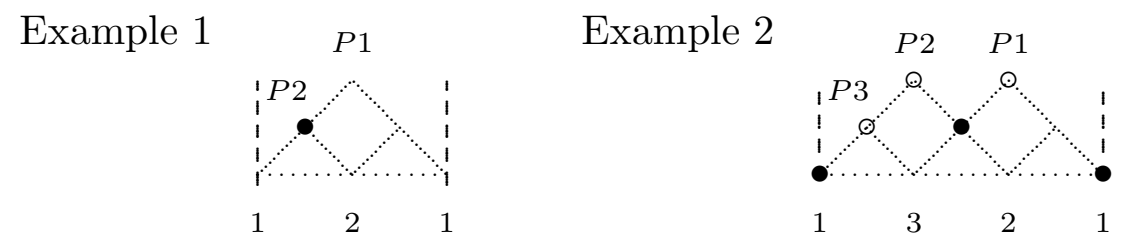

Example 1: Here is $\mathcal{R}_{0}$ the zero category, whereas there is an indecomposable module of the form $\Omega^{2} X$, namely the projective $P 2$.

In example 2, the indecomposable modules of the form $\Omega^{2} X$ are just 1 and $\operatorname{rad} P 1$ (marked by bullets). The subcategory $\mathcal{F}$ contains also $P 1$ and $P 2$, but not $P 3$. Note that $\mathcal{F}$ is closed under extensions, whereas the subcategories $\left\{\Omega^{2} X \mid X \in \bmod A\right\}$ and $\left\{P \oplus \Omega^{2} X \mid X \in \bmod A, P \in \operatorname{add}_{A} A\right\}$ are not closed under extensions (the extension closure of $\left\{\Omega^{2} X \mid X \in \bmod A\right\}$ is $\mathcal{F}$, and the extension closure of $\left\{P \oplus \Omega^{2} X \mid X \in\right.$ $\left.\bmod A, P \in \operatorname{add}_{A} A\right\}$ contains $3 \oplus P 2$.

Warning. Some mathematical papers (for example Auslander-Reiten [AR1], [AR2]) denote by $\Omega^{t}(\bmod A)$ the full subcategory of all modules of the form $P \oplus \Omega^{t} X$, with $P$ projective (and not, what one would expect, the full subcategory $\mathcal{C}$ of all modules of the form $\Omega^{t} X$, where $X$ is a module, or the additive closure of $\mathcal{C}$ ) and consider the question whether this subcategory $\Omega^{t}(\bmod A)$ is closed under extension or not. The examples above show that subcategories related to this subcategory may be closed under extensions whereas $\Omega^{t}(\bmod A)$ itself is not.

\section{Appendix C. Some canonical bijections.}

As an addition to appendix $\mathrm{B}$, we assume again that $A$ is a cyclic Nakayama algebra. We want to focus the attention to several further sets of modules which are in natural bijection to the subsets $\mathcal{S}, \mathcal{T}, \mathcal{U}$ mentioned above. The category $\mathcal{F}$ is defined by using as 
building blocks the modules $\Omega^{2} U$ with $U \in \mathcal{U}$, thus one may draw the attention also to the modules $\Omega U$ with $U \in \mathcal{U}$, we call them "valley" modules.

Recall from A.6 that an indecomposable module $V$ is a valley provided that $V=$ $\operatorname{rad} P=I / \operatorname{soc} I$, where $P$ is projective and $I$ is injective. Also, an indecomposable module $Z$ is a peak provided that $Z$ is both projective and injective. (Looking at the roof of the Auslander-Reiten quiver of $A$, we see that valleys and peaks alternate. Of course.)

An indecomposable projective module $P$ is said to be minimal provided that $\operatorname{rad} P$ is not projective. An indecomposable injective module $I$ is said to be minimal provided that $I / \operatorname{soc} I$ is not injective.

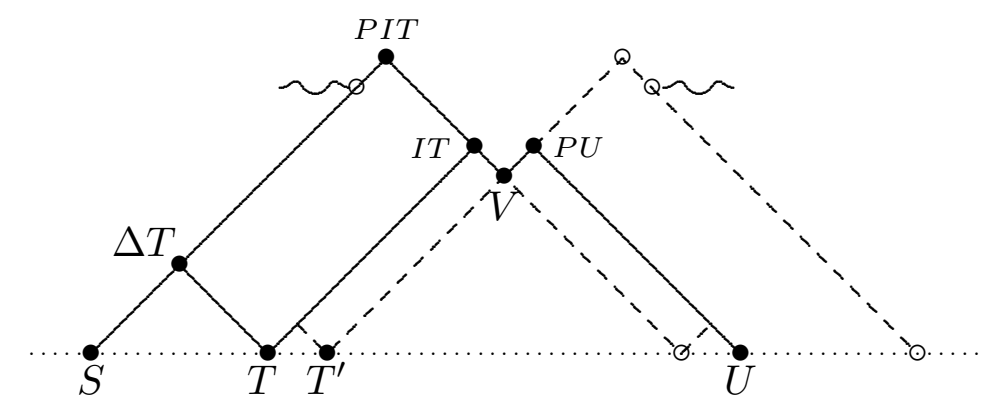

Some relevant bijections. We consider the modules marked above by bullets: these are modules which determine each other uniquely. Thus, we look at the set $\mathcal{T}$ of simple modules $T$ with id $T \geq 2$; the set $\mathcal{U}$ of simple modules $U$ with $\operatorname{pd} U \geq 2$; the set $\mathcal{S}$ of simple torsionless modules; the set of modules $\Delta T$ with $T \in \mathcal{T}$; the set of valley modules, the set of peak modules, the set of minimal injective modules and finally the set of minimal projective modules (the sets are arranged roughly in the same way as the modules are located in the Auslander-Reiten quiver): All these sets have the same cardinality, and any arrow in the following pictures exhibits a canonical bijection. Actually, the set $\mathcal{S}$ of simple torsionless modules appears twice in the picture, since two different bijections between $\mathcal{S}$ and $\mathcal{T}$ are relevant in our setting: if $T \in \mathcal{T}$, then, on one hand, the socle of $\Delta T$ is torsionless and simple, on the other hand, also $\tau^{-} T$ is torsionless and simple. These bijections are combined when we look at the Auslander-Reiten translation $\tau_{\mathcal{F}}$ of $\mathcal{F}$, see (4).

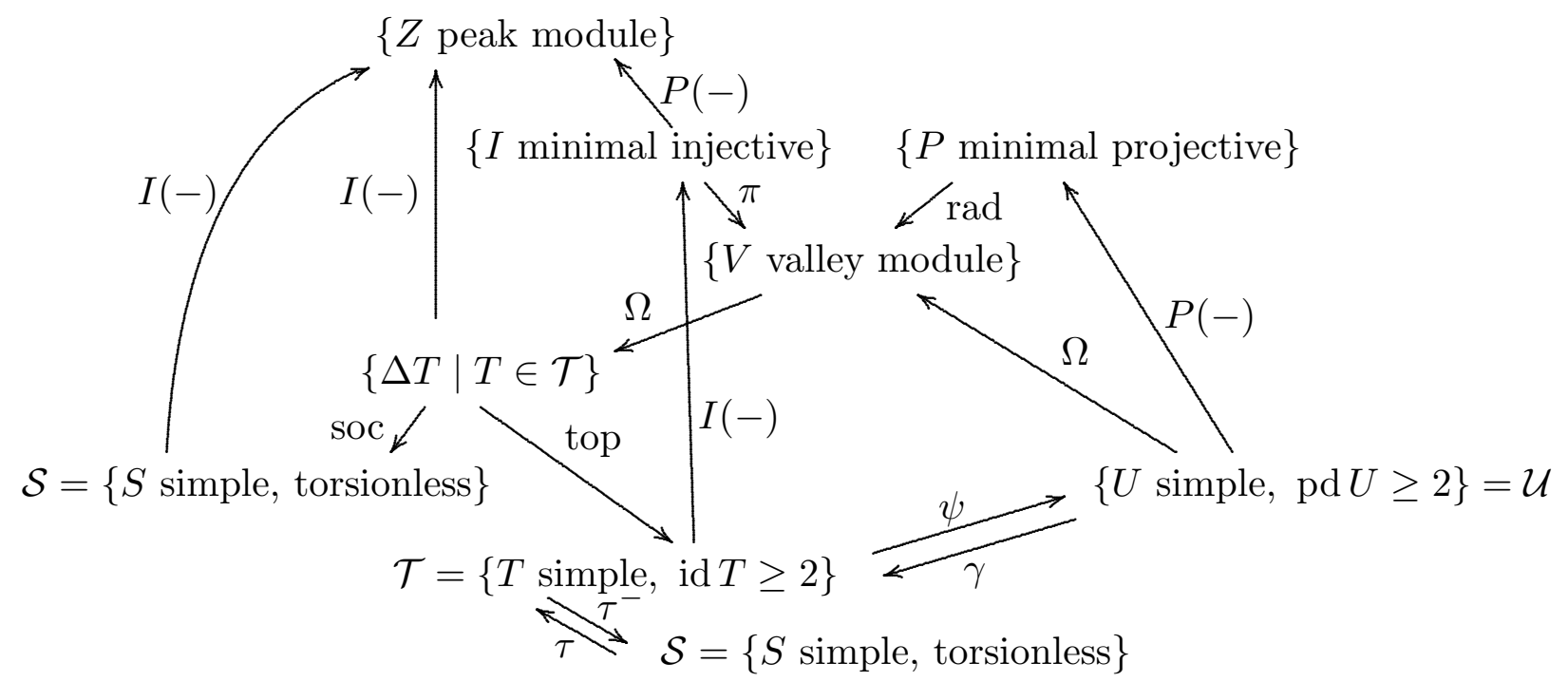


Here, $\pi(I)=I / \operatorname{soc} I$ for $I$ injective.

The display shows the interrelation between a large number of sets of cardinality $r$, where $|\mathcal{T}|=r$. However one should be aware that even this display is not yet complete. For example, similar to the torsionless simple modules, also the divisible simple modules play a role.

And, parallel to $\Delta T=\Omega V$, where $V$ is a valley, also the modules $\Sigma V$ should be taken into account. But we have refrained from doing so in order to keep the display manageable.

In general, if $\mathcal{W}$ is a set of simple modules and has cardinality $r$ (such as $\mathcal{S}, \mathcal{T}, \mathcal{U}$ or the set $\mathcal{D}$ of divisible modules), also the sets $\{P W \mid W \in \mathcal{W}\},\{\operatorname{rad} P W \mid W \in \mathcal{W}\}$, $\{I W \mid W \in \mathcal{W}\}$ and $\{I W / \operatorname{soc} \mid W \in \mathcal{W}\}$ are sets of cardinality $r$.

Minimal sets of relations. Let $r$ be the cardinality of $\mathcal{T}$. When looking at the large number of sets of modules which are in canonical bijection with $\mathcal{T}$, thus of cardinality $r$, one should be aware that it is another set of cardinality $r$ which really is the decisive one, namely any minimal set of relations which defines $A$. This is Sen's approach and it is definitely the relevant one. It leads immediately to an intrinsic choice of indices. Unfortunately, it seems that the use of these indices turns out to be not easy to digest, at least at a first reading. This is the reason that in contrast to Sen, we have started in a different way, namely with the set $\mathcal{T}$. As we saw, there is a canonical bijection between the set $\mathcal{T}$ and the set $\mathcal{V}$ of valleys $V$, and it is the set $\mathcal{V}$ which has to be considered as a natural setting for dealing with relations. We recall that relations can be, of course, interpreted as non-zero elements of $\mathrm{Ext}^{2}$, say of $\operatorname{Ext}^{2}(-,-)$. Any valley $V$ yields an exact sequence

$$
0 \rightarrow T \rightarrow I T \stackrel{f}{\rightarrow} P U \rightarrow U \rightarrow 0
$$

with $V=\operatorname{Im} f$. Its equivalence class in $\operatorname{Ext}^{2}(U, T)$ is non-zero. In this way, the set of valleys correspond to a minimal set of relations which defines $A$.

\section{Appendix D. Further invariants related to the finitistic dimension.}

In order to get hold of fin-pro $A$, Gélinas discusses in the paper [G1] not only del $S$, but also the grade of simple modules $S$ (thus the depth of $A$ ), and he looks for the numbers $t \geq 0$ such that $\mho^{t} \Omega^{t} S$ is torsionless. Examples of Nakayama algebras can be used in order to get a better understanding of these settings.

\section{D.1. The grade of a simple module and the depth of the algebra.}

Let $A$ be any artin algebra. Let $M$ be a module. The grade grade $M$ is defined as follows. We have grade $M=0$ iff $\operatorname{Hom}(M, A) \neq 0$, and grade $M=d \geq 1$ iff $\operatorname{Hom}(M, A)=$ $0, \operatorname{Ext}^{i}(M, A)=0$ for $1 \leq i<d$ and $\operatorname{Ext}^{d}(M, A) \neq 0$. The depth of $A$ is the maximum of grade $S$, where $S$ are the simple modules.

Proposition (Jans-Gélinas). Let $S$ be a simple module with finite grade $S=d \geq 1$. There are modules $N$ with injective dimension d which satisfy $\operatorname{Ext}^{d}(S, N) \neq 0$, for example $N=\tau \Omega^{d-1} S$. 
Proof. Let $P \bullet$ be a minimal projective resolution of $S$ and consider its truncation

$$
P_{d} \stackrel{f_{d}}{\longrightarrow} P_{d-1} \rightarrow \cdots \rightarrow P_{1} \stackrel{f_{1}}{\longrightarrow} P_{0} \rightarrow 0 .
$$

Here, $P_{0}=P(S)$ is a projective cover of $S$. For all $0 \leq i<d$, the cokernel of $f_{i+1}$ is $\Omega^{i} S$. In particular, $X=\Omega^{d-1} S$ is the cokernel of $f_{d}$. We form the $A$-dual

$$
P_{d}^{*} \stackrel{f_{d}^{*}}{\longleftarrow} P_{d-1}^{*} \leftarrow \cdots \leftarrow P_{1}^{*} \stackrel{f_{1}^{*}}{\longleftarrow} P_{0}^{*} \leftarrow 0 .
$$

This is an exact sequence, since $\operatorname{Ext}^{i}(S, A)=0$ for $0 \leq i<d$. By definition, $\operatorname{Tr} X$ is the cokernel of $f_{d}^{*}$. Thus, we have obtained a minimal projective resolution of the right module $\operatorname{Tr} X$

$$
0 \leftarrow \operatorname{Tr} X \leftarrow P_{d}^{*} \leftarrow P_{d-1}^{*} \leftarrow \cdots \leftarrow P_{1}^{*} \leftarrow P_{0}^{*} \leftarrow 0 .
$$

We apply $D$ to this sequence and get a minimal injective coresolution of $N=\tau \Omega^{d-1} S=$ $D \operatorname{Tr} \Omega^{d-1} S=D \operatorname{Tr} X$ with $D P_{0}^{*}=D P(S)^{*}=I(S)$. This shows that id $N=d$ and $\operatorname{Ext}^{d}(S, N) \neq 0$.

Corollary 1. For any simple module $S$, we have grade $S \leq \operatorname{del} S$.

Proof. This is trivially true if grade $S=0$. Thus we can assume that grade $S=d \geq 1$. The proposition asserts that there is a module $N$ with injective dimension $d$ which satisfies $\operatorname{Ext}^{d}(S, N) \neq 0$. According to Proposition 2.2, we get $d \leq \operatorname{del} S$.

\section{Corollary 2.}

$$
\operatorname{depth} A \leq \operatorname{del} A \text {. }
$$

\section{D.2. The case of Nakayama algebras.}

Lemma. Let $A$ be Nakayama and let $S$ be simple. Then $\operatorname{del} S=0$ iff grade $S=0$ iff $S$ is torsionless. Next, $\operatorname{del} S=1$ iff grade $S=1$ iff $S$ is not torsionless and $\operatorname{pd} S=1$. Also, del $S=2$ implies that grade $S=2$ (the converse is not rue, as we will see at the end of this section).

Proof. If $\Omega S$ is non-zero, then $\operatorname{Ext}^{1}(S, \Omega S) \neq 0$. Thus, if $\Omega S$ is non-zero and projective, then grade $S \leq 1$. Conversely, let grade $S=1$, and assume that $\Omega S$ is not projective. Then $\Omega S \rightarrow P(S) \rightarrow S$ is not an $\mho$-sequence, but this implies that $\operatorname{rad} P(S)=\Omega S \rightarrow P(S)$ is not an add $A$-approximation, thus $P(S)$ is not a minimal projective. But this implies that $\operatorname{rad} P(S)$ is projective, a contradiction.

Next, let del $S=2$. Then grade $S \geq 2$. But in general, grade $S \leq \operatorname{del} S$. Thus grade $S=2$.

Examples. There are cyclic Nakayama algebras of arbitrarily large finite global dimension with depth equal to 2 .

Let $m \geq 2$ and $n=2 m$. Let $A_{n}$ be the cyclic Nakayama algebra with Kupisch series $(2,3,3,4,4,5,5, \cdots, m+1, m+1, m+2)$. We denote the simple modules by $S_{i}$ with $1 \leq$ $i \leq n$, where $P\left(S_{1}\right)=2$ and $S_{i+1}=\tau^{-} S_{i}$. Thus, $\left|P\left(S_{2 i-1}\right)\right|=i+1$ and $\left|P\left(S_{2 i}\right)\right|=i+2$ 
for all $i \geq 1$. In particular, we see that $\operatorname{pd} S_{2 i}=1$ for all $i \geq 1$. Let us look at the simple modules with odd index. Since $\Omega S_{1}=S_{n}=S_{2 m}$ has projective dimension 1, we have $\operatorname{pd} S_{1}=2$. Since $\Omega^{2} S_{3}=S_{n}=S_{2 m}$ has projective dimension 1, we have pd $S_{3}=3$. For $i \geq 2$, we have $\Omega^{2} S_{2 i+1}=S_{i-1}$. This shows that $A_{n}$ has finite global dimension. Also,

$$
\operatorname{pd} S_{2^{t}-3}=2 t-2, \quad \text { for } \quad t \geq 2 \text {. }
$$

(The proof is by induction on $t \geq 2$. As we know, pd $S_{1}=2$. We have $\Omega S_{2^{t+1}-3}=$ $P\left(S_{2^{t+1}-4}\right) / \operatorname{soc}$ and $\operatorname{soc} P\left(S_{2^{t+1}-4}\right)=S_{2^{t}-3}$. By induction, we assume that $\mathrm{pd} S_{2^{t}-3}=$ $2 t-2$, therefore $\mathrm{pd} S_{2^{t+1}-3}=2+\operatorname{pd} S_{2^{t}-3}=2 t=2(t+1)-2$, as we want to show.) Thus the global dimension of $A_{n}$ gets arbitrarily large.

It remains to be seen that depth $A_{n}=2$. Since the simple modules with even index have projective dimension 1 , we have grade $S_{2 i} \leq 1$ for all $i$. We have $\Omega S_{2 i+1}=\operatorname{rad} P\left(S_{2 i+1}\right)=$ $P\left(S_{2 i}\right) /$ soc and there is a non-split exact sequence

$$
0 \rightarrow P\left(S_{2 i-1}\right) \rightarrow P\left(S_{2 i}\right) \oplus P\left(S_{2 i-1}\right) / \text { soc } \rightarrow P\left(S_{2 i}\right) / \text { soc } \rightarrow 0
$$

(an Auslander-Reiten sequence), thus

$$
\operatorname{Ext}^{2}\left(S_{2 i+1}, P\left(S_{2 i-1}\right)\right)=\operatorname{Ext}^{1}\left(P\left(S_{2 i}\right) / \text { soc, } P\left(S_{2 i-1}\right)\right) \neq 0 \text {. }
$$

This shows that grade $S_{2 i+1} \leq 2$ (and actually, grade $S_{n-1}=2$ ).

Here is the case $n=8$. On the left, there is the Auslander-Reiten quiver of $A_{8}$ with the function pd, and below the indices of the simple modules. On the right we highlight the module $S_{7}$ and the Auslander-Reiten sequence which shows that $\operatorname{Ext}^{2}\left(S_{7}, P\left(S_{5}\right)\right) \neq 0$.
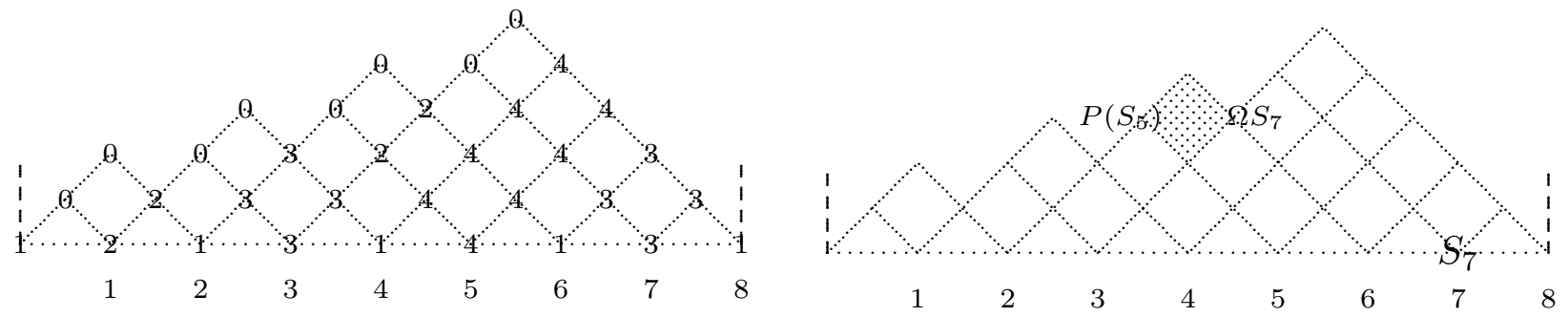

Already $A_{4}$ has a simple module $S$ with grade $S=2$ and $\operatorname{del} S=3$. Here is the algebra with the module $S=S_{3}$ encircled.

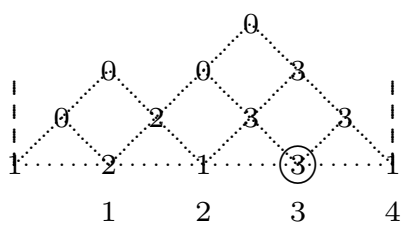

We have mentioned already that grade $S_{3}=2$. Its projective dimension is 3 , thus $\Omega^{2} S_{3}$ is not projective. On the other hand, since the global dimension of $A_{4}$ is 3 , all modules of the form $\Omega^{3} M$ are projective. Thus, we see that del $S_{3} \geq 3$ (and therefore $\operatorname{del} S_{3}=3$ ). 


\section{D.3. The modules $\mho^{t} \Omega^{t} S$.}

If $M$ is a module, let $\mho M=\operatorname{Tr} \Omega \operatorname{Tr} M$ (note that $\mho$ is a stable functor; it was considered by Auslander and Reiten in [AR], and is left adjoint to $\Omega$; one can show that $\mho M$ is just the cokernel of a minimal left add ${ }_{A} A$-approximation of $M$, see [RZ], 4.4).

If $S$ is a simple module, Gélinas looks for a non-negative number $t$ such that $\mho^{t} \Omega^{t} S$ is torsionless. Namely, Theorem 1.13 of Gélinas [Ge1] asserts: If $\mho^{t} \Omega^{t} M$ is torsionless, then $\operatorname{del} M \leq t$. There is the following immediate consequence (the "torsionfreeness criterion" 1.14): If there is $t \geq 0$ such that $\mho^{t} \Omega^{t} S$ is torsionless, then $\operatorname{del} A \leq \sup n_{S}$.

Here is an example of a cyclic Nakayama algebra and a simple module $S$ such that no module $\mho^{t} \Omega^{t} S$ with $t \geq 0$ is torsionless.
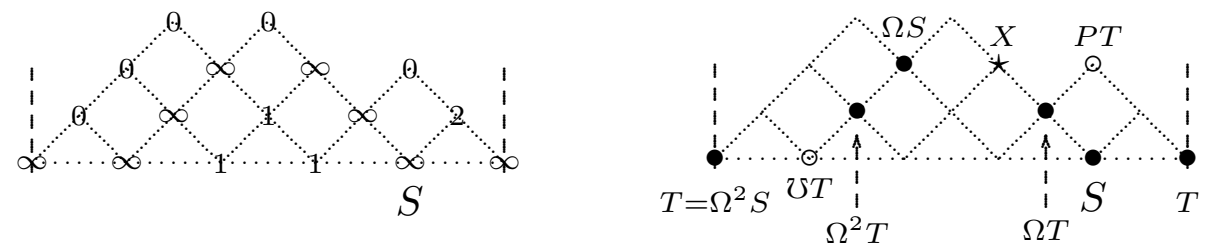

Claim. We have $\mho \Omega S=S$ and $\mho^{t} \Omega^{t} S=X$ for $t \geq 2$ (and both modules $S$ and $X$ are not torsionless). On the right, we have marked by bullets the modules $\Omega^{t} S$ with $0 \leq t \leq 4$ and stress that $\Omega^{5} S=\Omega^{2} S$. We write $T=\Omega^{2} S$. Also, we have inserted $X=\mho^{2} T$ as well as the projective cover $P T$ of $T$.

Proof. One checks immediately that $\mho \Omega \Omega S=S$. Thus, let us show that $\mho^{t} \Omega^{t} S=X$ for $t \geq 2$. It is easy to see that we have: (a) $\Omega^{3} T=T$, (b) $\mho \Omega T=T$, and (c) $\mho^{2} \Omega^{2} T=T$. We define (d) $X=\mho^{2} T$. Then we have (e) $\mho X=T$ (we stress that a minimal add $A$ approximation $X \rightarrow P T$ is not a monomorphism, but this does not matter!). It follows from (d) and (e) that we have (f) $\mho^{3} T=T$.

Now, let $t \geq 2$. If $t=3 s$ with $s \geq 1$, then

$$
\mho^{t} \Omega^{t} S=\mho^{2} \mho^{3(s-1)}(\mho \Omega) \Omega^{3(s-1)} T=X
$$

using (a), (b), (f) and (d). Next, for $t=3 s+1$ with $s \geq 1$, we get

$$
\mho^{t} \Omega^{t} S=\mho^{2} \mho^{3(s-1)}\left(\mho^{2} \Omega^{2}\right) \Omega^{3(s-1)} T=X
$$

using (a), (c), (f) and (d). Finally, for $t=3 s+2$ with $s \geq 0$, we get

$$
\mho^{t} \Omega^{t} S=\mho^{2} \mho^{3 s} \Omega^{3 s} T=X
$$

using (a), (f) and (d).

\section{References.}

[AR1] M. Auslander, I. Reiten. k-Gorenstein algebras and syzygy modules. J. Pure Appl. Algebra 92 (1994) 1-27. 
[AR2] M. Auslander, I. Reiten. Syzygy modules for noetherian rings. J. Algebra 183 (1996), $167-185$.

[B] H. Bass. Finitistic dimension and a homological generalization of semi-primary rings. Trans. Amer. Math. Soc. 95 (1960), 466-488

[Ga] P. Gabriel. The universal cover of a representation-finite algebra. In: Representations of Algebras. Springer Lecture Notes in Mathematics 903 (1981), 68-105.

[Ge] V. Gélinas. The depth, the delooping level and the finitistic dimension. arXiv:2004.04828 (2020).

[Gu] W. H. Gustafson. Global dimension in serial rings. Journal of Algebra, 97 (1985), $14-16$.

[M] D. Madsen. Projective dimensions and Nakayama algebras. Fields Institute Communications. 45. Amer. Math. Soc., Providence, RI, 2005. 247-265.

[N] T. Nakayama. On Frobeniusean algebras. I. Annals of Math. 40 (1939), 611-633.

[R1] C. M. Ringel. Representations of $k$-species and bimodules. J. Algebra 41 (1976), 269-302.

[R2] C. M. Ringel. The Gorenstein projective modules for the Nakayama algebras. I. Journal of Algebra (2013), 241-261. DOI 10.1016/j.jalgebra.2013.03.014

[RS] M. Rubey, C. Stump, et al. The Ringel permutation of the LNakayama algebra corresponding to a Dyck path. Mp00201. In: FindStat - The combinatorial statistics database (2020), www.findstat.org/Mp00201.

[Sh1] D. Shen. A note on resolution quivers. J. Algebra Appl. 13 (2014), 1350120.

[Sh2] D. Shen. The singularity category of a Nakayama algebra. J. Algebra 429 (2015), $1-18$.

[Sh3] D. Shen. A note on homological properties of Nakayama algebras. Archiv der Mathematik. 108 (2017), 251-261.

[S1] E. Sen. The $\phi$-dimension of cyclic Nakayama algebras. arXiv 1806.01449 (2018).

[S2] E. Sen. Syzygy filtrations of cyclic Nakayama algebras. arXiv 1903.04645 (2019).

[S3] E. Sen. Delooping level of Nakayama algebras. arXiv:2009.08888 (2020).

[S] J. P. Serre. Sur la dimension homologique des anneaux et des modules Noetheriens. Proceedings International Symposium on Algebraic Number Theory, Tokyo and Nikko (1955), 175-189.

[RZ] C. M. Ringel, P. Zhang. Gorenstein-projective and semi-Gorenstein-projective modules. Algebra \& Number Theory. Vol. 14 (2020), No. 1, 1-36.

DOI: $10.2140 /$ ant.2020.14.1

C. M. Ringel

Fakultät für Mathematik, Universität Bielefeld

POBox 100131, D-33501 Bielefeld, Germany

ringel@math.uni-bielefeld.de 